\title{
THE REPRESENTATION OF ABSTRACT INTEGRALS
}

BY

\section{DOROTHY MAHARAM}

Introduction. This paper is primarily concerned with linear order-preserving mappings from one function-space to another. For example, let $f$ be a (suitably restricted) function of two real variables, and consider the function $f^{\prime}(x)=\int f(x, y) d y$. The mapping $f \rightarrow f^{\prime}$ is a linear, countably additive mapping from the space of (suitably restricted) functions of two variables to the space of functions of one variable; and it is "order-preserving" in the sense that positive functions are mapped into positive functions. The main result of this paper implies that this example is (roughly speaking) typical; under mild hypotheses, mostly of countability, every linear, countably additive, order-preserving mapping $\phi$ from one function space $F_{0}$ to another, $F_{0}^{\prime}$, can be obtained by coordinatewise integration in a product space. That is, there exist spaces $X, Y$ such that $F_{0}$ is "isomorphic," in a sense to be defined below ( $\$ 1)$ to a certain space of functions on $X \times Y$, and $F_{0}^{\prime}$ is isomorphic to a space of functions on $X$, and under these isomorphisms $\phi$ corresponds to the mapping $f \rightarrow f^{\prime}$ where $f^{\prime}(x)=\int f(x, y) d y$, the integral being formed with respect to an ordinary (countably additive) $\sigma$-finite numerical measure on $Y$. (The exact theorem is stated in 5.3 below.) The formal properties of the mapping $\phi$, and the representation theorem just mentioned, entitle $\phi$ to be called an "abstract integral." The ordinary Lebesgue integral (over a fixed set with a $\sigma$-finite measure) is included as a special case, in which $F_{0}^{\prime}$ consists of the functions defined on a single point.

Abstract integrals have been considered from much this point of view before ${ }^{1}$ ), and representation theorems for linear mappings between two function spaces are known in many special cases $\left({ }^{2}\right)$. But the emphasis in the present investigation is on rather different aspects of the problem, and it is believed that there is little overlap with previous work. Briefly, the present treatment is aimed at saying as much as possible about the structure of a single "abstract integral" $\phi$, where $\phi$ is to have the salient features of the Lebesgue definite integral, but with values which (instead of being numbers) lie in as general a function space as can be handled. The only essential restriction we impose on our function spaces is that they satisfy the countable chain condition. Such spaces have no useful norms, and no norm conditions will be

Presented to the Society, September 5, 1953; received by the editors August 19, 1952.

(1) See [2, Chapter III], and references given there.

$\left({ }^{2}\right)$ See [3], and references there given. 
imposed on $\phi$, their role being taken over by the requirement of "positivity" (i.e., that $\phi$ preserve order) $\left(^{3}\right)$.

We shall allow the functions considered to be infinite, and a finite function may have a (wholly or partly) infinite function as its "integral." This is only partly for the sake of generality; the main reason is that, even if we restricted attention to finite functions to begin with, infinite functions would in general be introduced by the constructions. However, the extra generality seems desirable, even in the Lebesgue case; and, though infinities cause some complications, they also lead to some compensating simplifications of the technique (particularly in $\$ \S 6$ and 7 ). Thus the situation we actually consider is somewhat more general than that described in the first paragraph. The "abstract integral" $\phi$ is now a mapping of a certain subclass $G$ of one "extended" function space $F$ (extended by infinite functions) in another, $F^{\prime}$; the set-up adopted $(\$ 2)$ is a natural generalization of that for the ordinary Lebesgue integral, $G$ then corresponding to the class of functions which are "integrable" in the sense of [9] (i.e., for which $\int f(x) d x$ is unambiguous, finite or not). It suffices, however, to assume only that $\phi$ is defined on a much smaller class $G_{0}$ of functions (see 2.2), as $\phi$ can then be extended to a suitable $G$. For example, let $F_{0}$ and $F_{0}^{\prime}$ denote the sets of finite functions in $F$ and $F^{\prime}$ respectively; then any linear, countably additive, order-preserving mapping $\phi$ of $F_{0}$ in $F_{0}^{\prime}$ can be extended to one of a suitable $G$ in $F^{\prime}$, and we recover the special case, discussed in the first paragraph, in which $\phi$ maps one space of finite functions in another.

The methods used are direct and are largely based on the author's theory of abstract-valued measures [5]. The notations follow [5] as far as possible. The basic definitions and preliminaries are dealt with in $\$ \$ 1-3$, which in particular contain the notions of (abstract) function space, and of isomorphism and strict isomorphism of function spaces, and establish the relations between function-spaces and Boolean $\sigma$-algebras, and between generalized "integrals" and the corresponding generalized "measures." The main step in deriving the principal theorem (Theorem 8, 5.3) is then the result (Theorem 6, 5.1) that the generalized measure algebras which arise in this way can be imbedded in others (studied in \$4) which satisfy the additional postulates required of "abstract measure algebras" in [5]. Theorem 6, besides extending the scope of the theory in [5], then enables that theory to be applied to determine the structure of the abstract integral. In Theorem 9 (6.1) a more precise result is obtained, under stronger hypotheses; and it is finally shown (Theorem 10, 7.2) that, under intermediate hypotheses, the abstract integral can be replaced by one to which the stronger hypotheses (and so Theorem 9) apply.

( $\left.{ }^{3}\right)$ The results can be extended to mappings $\phi$ which, instead of preserving order, satisfy the weaker condition of "regularity" [3], and one can further give simultaneous representations for certain families of regular $\phi$ 's. It is hoped that details will appear elsewhere. 
One application of these results is worth remarking, though we shall not enter into it in detail. Since every vector $\sigma$-lattice can be imbedded in a function space $\left({ }^{4}\right)$, our results give in particular an analysis of the structure of an arbitrary (order-preserving) homomorphism of one vector $\sigma$-lattice, satisfying the countable chain condition, in another.

\section{FunCTION SPACES, ALGEBRAS AND ISOMORPHISMS}

1.1. The basic type of function space $F$ adopted in the sequel is an abstraction from the space of all measurable real functions, finite or infinite, on a measure space $S$, two functions being identified if they differ only on a null set. We shall later require $F$ to satisfy the countable chain condition (see 1.8), which corresponds to requiring the measure on $S$ to be $\sigma$-finite; but this will not be needed for most of the results in the present section.

Other types of function space are conceivable, but it is not necessary to consider them explicitly, as their theory is included in that of the type adopted here. For any function space with which we could be concerned would certainly be a vector $\sigma$-lattice (satisfying the countable chain condition); now it follows from known results $[1 ; 7]$ that every such lattice $V$ can be imbedded in a function space of our basic type (also satisfying the countable chain condition) in such a way that (in view of 2.2 below) any "abstract integral" $\phi$ on $V$ could be extended to one on $F\left({ }^{5}\right)$.

Most of the contents of $\S 1$ are essentially known (see e.g. [7]), but are formulated here to establish the notation and in a form convenient for what follows.

1.2. Let $S$ be an arbitrary non-empty set, $\mathcal{B}$ a Borel field of subsets $B$ of $S$, and $\mathcal{N}$ a $\sigma$-ideal of subsets of $S$; the sets of $B$ may be thought of as "measurable," and those of $\mathcal{N}$ as "null," though there is, of course, no actual measure involved in general. We shall usually assume (without loss of generality) that $B$ and $\mathcal{N}$ are "complete"-i.e., $\mathcal{N} \subset \mathcal{B}$ and if $N_{2} \subset N_{1} \in \mathcal{N}$, then $N_{2} \in \mathcal{N}$. We shall use the term "function" as an abbreviation for a function $f$ on $S$ whose values are real numbers or $\pm \infty$ (i.e., $-\infty \leqq f(s) \leqq \infty$, for all $s \in S$ ), and which is "measurable $B$ "-i.e., for each (finite) real number $\rho$, the set $X_{\rho}(f)=\{s \mid f(s)<\rho\}$ is in $B$. The set of all functions forms the "realized function space" $\mathcal{F}(S, \mathcal{B}, \mathcal{N})$, or $\mathcal{F}$ for short. Two functions $f, g$ are equivalent if there exists a set $N \in \mathcal{N}$ such that $f(s)=g(s)$ whenever $s \in S-N$. The equivalence classes $\{f\}$ of functions form the function space $F(S, \mathscr{B}, \mathcal{N})$, or $F$. To simplify the notation, however, we shall usually omit the brackets from $\{f\}$, using $f$ to denote both a function and its equivalence class.

(4) See [7] and $\$ 1$ below.

(5) More precisely, $V$ is a restricted direct sum of a sequence $\left\{V_{n}\right\}$ of vector $\sigma$-lattices, satisfying the countable chain condition, each of which has a unit element; and each $V_{n}$ can be realized by a class $G_{n}$ of finite functions, which contains all bounded functions, in a function space $F_{n}$ of our type. Thus $V$ is imbedded in the direct sum $F$ of the $F_{n}$ 's, which is also a function space of our basic type, and the union $G_{0}$ of the $G_{n}$ 's satisfies the requirements of 2.2 below. 
1.3. It is easy to see that, with the usual definitions of sum, product and ordering of functions, the usual rules of analysis hold in $\mathcal{F}$ and so in $F$, except for the complication caused by the fact that the values $+\infty,-\infty$ cannot be added. We make, however, the convention that $\infty \cdot 0=0 \cdot \infty=0$. Thus, for example: (a) if $f \in \mathcal{F}(S, \mathbb{B}, \mathcal{N})$ and $\Lambda$ is any Borel set of real numbers, $f^{-1}(\Lambda) \in \mathcal{B}$, (b) if $f \in F$, and $\rho$ is any real number, or $\pm \infty$, then $\rho f \in F$, (c) if $f, g \in F$ and if $f+g$ exists (i.e., if the sets $\{s \mid f(s)=\infty=-g(s)\}$ and $\{s \mid-f(s)$ $=\infty=g(s)\}$ are both in $\mathcal{N})$, then $f+g \in F$. In particular, the finite functions in $F$ form a linear space. Again, if $f_{n} \in F(n=1,2, \cdots)$, then sup $f_{n}$ (i.e., the equivalence class of the function whose value at each $s \in S$ is $\sup f_{n}(s)$ ), $\inf f_{n}$, and (if they exist) $\lim f_{n}$ and $\sum f_{n}$, all belong to $F\left(^{(}\right)$.

We write $f \leqq g$, where $f, g \in F$, to mean that $f(s) \leqq g(s)$ for all $s \in S-N$ where $N \in \mathcal{N} ; f<g$ means that $f \leqq g$ and $\{f\} \neq\{g\}$; and $f \ll g$ means that $f(s)<g(s)$ for all $s \in S-N(N \in \mathcal{N})\left({ }^{7}\right)$. We denote the constant function whose value is always $c$ (where $-\infty \leqq c \leqq+\infty$ ) by $c$ also, and write $\sup (f, 0)=f^{+}$, $\sup (-f, 0)=f^{-}$. Thus $0 \leqq f^{+}, 0 \leqq f^{-}$, and $f^{+}-f^{-}$exists and equals $f$.

For each $f \in \mathcal{F}$ we define the "locus" $[f]$ to be the set $\{s \mid f(s) \neq 0\} \in \mathcal{B}\left({ }^{8}\right)$. If $f \in F,[f]$ is defined similarly; it is determined only to within a null set.

For each $B \in \mathcal{B}, \chi_{B}$ denotes the characteristic function of $B$; that is, $\chi_{B}(s)=1$ if $s \in B, 0$ if $s \in S-B$. To facilitate printing, however, we shall write $\chi_{B}$ as $\chi(B)$ whenever possible. We also use $\chi(B)$ to denote the equivalence class (modulo $\mathcal{N}$ ) of this characteristic function in $F$. Clearly $[\chi(B)]$ $=B(\bmod \mathcal{N}), \chi(S)=1$ (the "unit function"), and $\chi(0)=0$.

1.4. Let $B_{1}, B_{2}, \cdots, B_{n}$ be $\operatorname{disjoint}\left({ }^{9}\right)$ sets of $\mathcal{B}$ whose union is $S$, and let $\rho_{1}, \rho_{2}, \cdots, \rho_{n}$ be (finite) real numbers; then the sum $\sum_{1}^{n} \rho_{i} \chi\left(B_{i}\right)$ (in $\mathcal{F}$ or $F$ ) is called a "step-function." It is easy to see that the sum and product of finitely many step-functions are step-functions.

Lemma 1. Given $f \in \mathcal{F}$, there exist (a) a sequence $B_{n}$ of sets of $\mathrm{B}$, and (b) a sequence $\rho_{n}$ of rational numbers, such that $\sum_{1}^{\infty} \rho_{n} \chi\left(B_{n}\right)$ exists (finite or infinite) and equals $f$ (for all $s \in S$ ).

Suppose first that $0 \leqq f \leqq 1$. Let $B_{1}=\left\{s \mid f(s) \geqq 2^{-1}\right\}$; then $2^{-1} \chi\left(B_{1}\right) \leqq f$ $\leqq 2^{-1} \chi\left(B_{1}\right)+2^{-1}$. An easy induction argument gives sets $B_{2}, \cdots, B_{n} \in \mathcal{B}$ such that $\sum_{1}^{n} 2^{-m} \chi\left(B_{m}\right) \leqq f \leqq \sum_{1}^{n} 2^{-m} \chi\left(B_{m}\right)+2^{-n}$, and the result follows. It is easy to derive the general case from this one.

COROLLARY. Every element of $F$ is a limit of a sequence of step-functions.

(") If $f_{n} \in F$, we say (for example) that " $\sum f_{n}$ exists" if, for each $s \in S-N$ (where $N \in \mathcal{N}$ ), the sequence of numbers $f_{1}(s)+f_{2}(s)+\cdots+f_{n}(s)$ tends to a limit (possibly infinite) as $n \rightarrow \infty$.

(7) Note that in [3], for example, the symbol " $f<g$ " is used to mean " $f \ll g$ " in the present notation. Note also that " $f \ll g$ " does not mean that $f$ is uniformly below $g$.

(8) This use of [ ] departs from the notation in [5].

( ${ }^{\circ}$ Throughout this paper, "disjoint" means "pairwise disjoint." 
REMARK. If $f \geqq 0$, the constants $\rho_{n}$ in Lemma 1 can be taken to be nonnegative.

1.5. Isomorphisms. We define two function spaces $F=F(S, \mathcal{B}, \mathcal{N})$ and $F^{\prime}=F\left(S^{\prime}, \mathcal{B}^{\prime}, \mathcal{N}^{\prime}\right)$ to be isomorphic under $\psi$ if $\psi$ is a 1-1 mapping of $F$ onto $F^{\prime}$ which preserves linear operations, limits, and order. That is, we require: $\sum_{1}^{\infty} \psi\left(f_{n}\right)$ exists if and only if $\sum_{1}^{\infty} f_{n}$ exists, and if both exist then $\sum \psi\left(f_{n}\right)$ $=\psi\left(\sum f_{n}\right)$; with similar statements for multiplication of $f$ by a (possibly infinite) constant, $\lim f_{n}, \sup f_{n}, \inf f_{n}$, and the relations $\leqq,<$, and «. It will follow that $\psi(0)=0$, and that $\psi(f)$ is finite if and only if $f$ is finite. It is not required that $\psi(f g)=\psi(f) \psi(g)$.

Lemma 2. A 1-1 mapping $\psi$ of $F$ onto $F^{\prime}$ is an isomorphism provided that, for all $f, g \in F:$

(1) $\psi(f)+\psi(g)$ exists if and only if $f+g$ exists, and if both exist then $\psi(f+g)$ $=\psi(f)+\psi(g)$.

(2) $\psi(f) \geqq 0$ if and only if $f \geqq 0$.

Proof. Suppose $\psi$ (and thus also $\psi^{-1}$ ) satisfies these requirements. Then, from (1) applied to the functions $\psi^{-1}(0)$ and 0 , we have $\psi(0)=0$. Again, applying (1) to the functions $f$ and $-f$, we obtain:

(3) If $f$ is finite, then so is $\psi(f)$, and then $\psi(-f)=-\psi(f)$.

We shall deduce that $\psi(-f)=-\psi(f)$ even if $f$ is infinite. First note:

(4) If $-\infty \ll f \leqq g, \psi(f) \leqq \psi(g)$.

For we can then write $g=f+h$ where $h \geqq 0$, and (4) follows from (1) and (2). Now let $B$ be any set in $B$, and write $\psi(\chi(B))=f^{\prime}$; from (4) we obtain $\psi(\chi(B)) \geqq \psi(n \chi(B))=n f^{\prime}$, from (1), where $n=1,2, \cdots$, and it follows that $\psi(\infty \chi(B)) \geqq \infty f^{\prime}$. But a similar argument applied to $\psi^{-1}$ gives $\infty \chi(B)$ $\leqq \psi^{-1}\left(\infty f^{\prime}\right)$, and so (from (4) again) $\psi(\infty \chi(B)) \leqq \infty f^{\prime}$. Hence $\psi(\infty \chi(B))=\infty f^{\prime}$. A similar argument gives $\psi(-\infty \chi(B))=-\infty f^{\prime}=-\psi(\infty \chi(B))$. Now any $f \in F$ can be written in the form $f=g+\infty \chi\left(B_{1}\right)+(-\infty) \chi\left(B_{2}\right)$, where $g$ is finite and $[g], B_{1}, B_{2}$ are disjoint; hence

$$
\psi(-f)=-\psi(f)
$$

(all $f \in F)$.

We can now improve (4) to

$\left(4^{\prime}\right)$ If $f \leqq g, \psi(f) \leqq \psi(g)$.

For (4) gives $\psi\left(f^{+}\right) \leqq \psi\left(g^{+}\right)$and $\psi\left(f^{-}\right) \geqq \psi\left(g^{-}\right)$, and the result now follows from $\left(3^{\prime}\right)$ and (1). Thus $\psi$ preserves (arbitrary) suprema and infima. Again, if $f \gg 0$, then $\psi(f) \gg 0$. For we know $\psi(f)>0$; suppose $\psi(f)=0$ on a set $B^{\prime} \in \mathcal{B}^{\prime}$, where $B^{\prime}$ is not in $\mathcal{N}^{\prime}$, and define $g=\psi^{-1}\left(\chi\left(B^{\prime}\right)\right), h=\inf (f, g)$. Then $\psi(h)=0$, and so $h=0$; but since $f \gg 0$ and $g>0$ it follows that $h=\inf (f, g)>0$, giving a contradiction. The relation $f \ll g$ is equivalent (even for infinite functions) to $g-f \gg 0$, and hence is also preserved.

From (1) and $\left(3^{\prime}\right)$ we easily see that $\psi(\rho f)=\rho \psi(f)$ whenever $\rho$ is rational. Thus the whole lemma will readily follow once it is shown that $f_{n} \rightarrow f$ implies 
$\psi\left(f_{n}\right) \rightarrow \psi(f)$. This is clearly the case if the sequence $f_{n}$ is monotonic (for the limit is then $\sup f_{n}$ or $\left.\inf f_{n}\right)$; and this shows that $\psi\left(\lim \sup f_{n}\right)=\lim \sup \psi\left(f_{n}\right)$, and similarly for $\lim$ inf. But, generally, $f_{n} \rightarrow f$ if and only if $\lim \sup f_{n}=f$ $=\lim \inf f_{n} ;$ and the lemma follows.

1.6. Strict isomorphisms. An isomorphism $\psi$ of $F=F(S, B, \mathcal{N})$ onto $F^{\prime}$ $=F\left(S^{\prime}, \mathbb{B}^{\prime}, \mathcal{N}^{\prime}\right)$ will be called strict if $\psi(1)=1^{\prime}$, the unit function in $F^{\prime}$. Let $E$ and $E^{\prime}$ denote the Boolean $\sigma$-algebras $B \bmod \mathcal{N}, B^{\prime} \bmod \mathcal{N}^{\prime}$, respectively. We shall prove:

THEOREM 1. The following statements are equivalent:

(i) The function spaces $F$ and $F^{\prime}$ are isomorphic.

(ii) The algebras $E$ and $E^{\prime}$ are ( $\sigma$-)isomorphic.

(iii) $F$ and $F^{\prime}$ are strictly isomorphic.

To prove that (i) implies (ii), let $\psi$ be an isomorphism of $F$ onto $F^{\prime}$; since the relation $\ll$ is preserved, we have

$$
0 \ll \psi(1) \ll \infty .
$$

Now we remark that, if $f, g \in F$ and $0 \leqq g \ll \infty$, then a necessary and sufficient condition that $f=g \chi(B)$ for some $B \in B$ is that $0 \leqq f \leqq g$ and inf $(f, g-f)$ $=0$. A similar statement applies, of course, to $F^{\prime}$. Hence, for each $B \in \mathcal{B}$, we have $\left.\psi(\chi(B))=\psi(1) \chi\left(B^{\prime}\right)\right)$ for some $B^{\prime} \in B^{\prime}$, say $B^{\prime}=\theta(B)$. From (5), $\theta(B)$ is unique, modulo $\mathcal{N}^{\prime}$, when $B$ is given modulo $\mathcal{N}$; thus $\theta$ maps $E$ in $E^{\prime}$. We shall show that, for any $f \in F$ and $B \in \mathcal{B}$,

$$
\psi(f \chi(B))=\psi(f) \chi(\theta(B)) .
$$

In fact, if $f \geqq 0$ this follows from the relation $f \chi(B)=\inf (f, \infty \chi(B))$; and, applying this to $f^{+}$and $f^{-}$separately, we see that (6) holds for all $f \in F$.

It is now easy to verify, using (6), that $\theta$ is an isomorphism of $E$ onto $E^{\prime}$.

Since (iii) implies (i) trivially, we have only to show that (ii) implies (iii). A preliminary construction is necessary. By a "spectrum" on $S\left({ }^{10}\right)$ we shall mean a mapping from the rational numbers $\rho$ to sets $X_{\rho} \in \mathcal{B}$ such that: (a) $\rho<\sigma$ implies $X_{\rho} \subset X_{\sigma}$, (b) $\cup\left\{X_{\rho} \mid \rho<\sigma\right\}=X_{\sigma}$. Each $f \in \mathcal{F}(S, \mathcal{B}, \mathcal{N})$ determines such a spectrum by the rule $X_{\rho}(f)=\{s \mid f(s)<\rho\}$. Conversely (see $[10 ; 8]$ ) each spectrum $\left\{X_{\rho}\right\}$ arises in this way from a unique $f \in \mathcal{F}$. If two functions $f, g$ are equivalent modulo $\mathcal{N}$, their spectra $\left\{X_{\rho}(f)\right\},\left\{X_{\rho}(g)\right\}$ are also equivalent, in the sense that for each $\rho$ the symmetric difference of $X_{\rho}(f)$ and $X_{\rho}(g)$ is in $\mathcal{N}$. The converse is also immediate, so there is a 1-1 correspondence between elements of $F(S, \mathcal{B}, \mathcal{N})$ and equivalence classes of spectra mod $\mathcal{N}$.

Now let $\theta$ be an isomorphism of $E$ onto $E^{\prime}$. Clearly $\theta$ maps spectra $(\bmod \mathcal{N})$ on spectra $\left(\bmod \mathcal{N}^{\prime}\right)$, and so induces a 1-1 mapping $\psi$ of $F$ onto $F^{\prime}$; and it is easy to verify that $\psi$ has properties (1) and (2) of Lemma (2) (1.5), and that $\psi(1)=1^{\prime}$, so that $\psi$ is a strict isomorphism.

(10) See [10] and [8]. 
CoROLlaRy 1. Every strict isomorphism of $F$ is induced by an isomorphism $\theta$ of $E$, as above. The most general isomorphism of $F$ is obtained from a strict isomorphism by multiplying all functions by a fixed function $f_{0}$ such that $0 \ll f_{0} \ll \infty$.

For if $\psi_{1}$ is a strict isomorphism of $F, \psi_{1}$ induces an isomorphism $\theta$ of $E$ as above, and $\theta$ induces a strict isomorphism $\psi_{2}$ of $F$. Then $\psi_{2}^{-1} \psi_{1}$ is a strict isomorphism of $F$ onto itself which leaves $E$ invariant, in the sense that it maps every $\chi(B)$ onto itself, and from Lemma $1(1.4)$ it must be the identity mapping; that is, $\psi_{1}=\psi_{2}$.

If $\psi$ is an arbitrary isomorphism of $F$, we have seen that $0 \ll \psi(1) \ll \infty$; the correspondence $\psi_{1}$ defined by $\psi_{1}(f)=\psi(f) / \psi(1)$ is evidently a strict isomorphism, and $\psi(f)=\psi(1) \psi_{1}(f)$. (Similarly $\psi(f)=\psi_{2}\left(f / \psi^{-1}\left(1^{\prime}\right)\right.$ ), where $\psi_{2}$ is another strict isomorphism.)

Incidentally the argument shows there is a 1-1 correspondence between isomorphisms of $E$ and strict isomorphisms of $F$ given by the constructions used in the proof of Theorem 1.

Corollary 2. An isomorphism $\psi$ of $F$ onto $F^{\prime}$ is strict if and only if $\psi(f g)$ $=\psi(f) \psi(g)($ for all $f, g \in F)$.

If $\psi(1)=1^{\prime},(6)$ above gives $\psi(\chi(B))=\chi(\theta(B))$, and from Lemma 1 (1.4) we have $\psi(f) \psi(g)=\psi(f g)$ provided $f, g \geqq 0$; but the general case follows at once on considering $f^{+}, f^{-}$and $g^{+}, g^{-}$, separately. The converse implication follows trivially, in view of (5).

1.7. In what follows, we shall usually not distinguish between strictly isomorphic function spaces; thus $F(S, \mathcal{B}, \mathcal{N})$ is determined by the isomorphism class of the Boolean $\sigma$-algebra $E=\mathbb{B} / \mathcal{N}$ alone, and we shall accordingly write $F(S, B, \mathcal{N})$ henceforth as $F(E)$. It is understood that in applying any isomorphism to $F(E)$, we also apply the induced isomorphism (Theorem $1)$ to $E$. Thus if $x \in E$, we can define $\chi(x) \in F(E)$ to be the characteristic function of any of the equivalent sets $B$ corresponding to $x$ in any particular realization involved, and have that the relation between $x$ and $\chi(x)$ is preserved by strict isomorphism. Similarly, if $f \in F(E)$, we define its "locus" in $E$, also denoted by $[f]$, to be the element of $E$ corresponding to the set $\{s \mid f(s) \neq 0\}$, modulo $\mathcal{N}$, in any realization; the relation between $f$ and $[f]$ is preserved by (not necessarily strict) isomorphism.

1.8. The countable chain condition. Throughout what follows, all function spaces considered will be assumed to satisfy:

(7) Every strictly increasing transfinite sequence $f_{1}<f_{2}<\cdots<f_{\alpha}<\cdots$ of elements of $F$ must terminate countably.

It is easy to see that $F(E)$ satisfies (7) if and only if $E$ satisfies the corresponding "countable chain condition":

(8) Every disjoint collection of nonzero elements of $E$ is countable. 
In fact, (7) implies (8) trivially; conversely, if (8) holds, we may suppose (by a preliminary change of scale) that the functions in (7) all satisfy $-1 \leqq f$ $\leqq 1$, and then the argument is similar to that in $[5$, p. 288].

An immediate consequence of (7) and (8) is that $F$ is a complete lattice: given any nonempty subset $V \subset F$, the elements sup $\{f \mid f \in V\}$ and inf $\{f \mid f \in V\}$ exist, and moreover there exists a sequence $f_{n} \in V(n=1,2, \cdots)$ such that $\sup \{f \mid f \in V\}=\sup f_{n}$, and similarly for infima.

1.9. It will sometimes be desirable to use concrete realizations for $E$ and $F$; one, which will be particularly convenient later, is the "representation space realization," obtained by taking $S=$ representation space of $E, \mathcal{N}$ $=$ family of sets of the first category, and $B=$ family of sets each differing from an open-closed set by a set of first category at most. Since the countable chain condition holds, we may take $F=$ class of continuous functions on $S$, modulo $\mathcal{N}$, the values $\pm \infty$ being allowed (cf. $[5, \S 4]$ ). It will then be convenient to represent each $f \in F$ by the (unique) continuous function in its equivalence class. Thus, for example, $\sum_{1}^{\infty} f_{n}$ will be represented, not in general by the pointwise sum of the functions $f_{n}$, but by the continuous function which differs from this sum on a first category set (at most).

\section{INTEGRALS}

2.1. Let $F=F(E)$ and $F^{\prime}=F\left(E^{\prime}\right)$ be two function spaces (satisfying the countable chain condition). Roughly speaking, an "integral" will be a linear order-preserving mapping of $F$ in $F^{\prime}$; but some complications arise from the fact that functions can be infinite. First let $\phi_{1}$ be a mapping of the nonnegative functions of $F$ in the non-negative functions of $F^{\prime}$ such that

( $\alpha) \phi_{1}\left(\sum f_{n}\right)=\sum \phi_{1}\left(f_{n}\right)(n=1,2, \cdots)$,

( $\beta)$ There exist elements $g_{1}, g_{2}, \cdots$, of $F$ such that $\sum g_{n} \gg 0, g_{n} \geqq 0$, and $\phi_{1}\left(g_{n}\right) \ll \infty(n=1,2, \cdots)\left({ }^{11}\right)$.

It follows that $\phi_{1}(0)=0$. Let $G$ be the set of all functions $f \in F$ for which $\phi_{1}\left(f^{+}\right)-\phi_{1}\left(f^{-}\right)$exists (i.e., for which inf $\left.\left\{\phi_{1}\left(f^{+}\right), \phi_{1}\left(f^{-}\right)\right\} \ll \infty\right)$, and define $\phi(f)=\phi_{1}\left(f^{+}\right)-\phi_{1}\left(f^{-}\right)$whenever $f \in G$. Clearly $G$ contains all non-negative (and also all nonpositive) functions in $F$, and $\phi$ agrees with $\phi_{1}$ on the non-negative functions. It is easy to see that the following properties hold.

(1) If $f \in G$ and $f \geqq 0$, then $f \in G$ and $\phi(f) \geqq 0$.

(2) $f \in G$ if and only if $\phi\left(f^{+}\right)-\phi\left(f^{-}\right)$exists.

(3) If $f \in G$ and $\rho$ is real (and finite), $\rho f \in G$ and $\phi(\rho f)=\rho \phi(f)$.

(4) If $f, g \in G$ and $f+g, \phi(f)+\phi(g)$ both exist, then $f+g \in G$ and $\phi(f+g)$ $=\phi(f)+\phi(g)$.

(5) If $f_{n} \geqq 0(\dot{n}=1,2, \cdots)$, then $\phi\left(\sum f_{n}\right)=\sum \phi\left(f_{n}\right)$.

(11) Instead of $(\beta)$, it might seem more natural to require that there exists $g \gg 0$ in $F$ such that $\phi_{1}(g) \ll \infty$. But this requirement is definitely stronger than $(\beta)$ in general, though it is equivalent to $(\beta)$ in many cases, e.g., if $E^{\prime}$ is a measure algebra or if $\phi_{1}$ is "homogeneous" (see $\S 7$ ). 
(6) The unit element $e$ of $E$ can be expressed as $e=V x_{n}\left(x_{n} \in E\right.$, $n=1,2, \cdots)$ in such a way that $\phi\left(\chi\left(x_{n}\right)\right) \ll \infty\left({ }^{12}\right)$.

(Since (6) follows from $(\beta)$, in view of Lemma $1,1.4$, the only property requiring proof here is (4). Now (4) holds trivially if $f, g \geqq 0$, and can then be deduced when $f \leqq 0 \leqq g$; the general case then follows on considering the functions $f^{+}+g^{+}$and $f^{-}+g^{-}$.)

Conversely, any mapping $\phi$ of a subset $G$ of $F$ in $F^{\prime}$ which satisfies (1)-(6) will clearly arise in this way from $\phi_{1}=\phi$ restricted to non-negative functions, and $\phi_{1}$ satisfies $(\alpha)$ and $(\beta)$. If $\phi$ (or, equivalently, $\phi_{1}$ ) satisfies the two further requirements:

(7) If $f>0$, then $\phi(f)>0$;

(8) $\phi(1) \gg 0$,

we say that $\phi$ is an $F^{\prime}$-integral on $F$, with $G$ as its set of integrable functions. Conditions (7) and (8) involve no real loss of generality. If (7) does not hold, we have only to enlarge the class $\mathcal{N}$ of null sets (in any realization of $F$ as $F(S, \mathcal{B}, \mathcal{N}))$ to include all sets $B$ for which $\phi(\chi(B))=0$; in effect this amounts to replacing $S$ by a subspace, or equivalently to replacing $E$ by a suitable principal ideal. Similarly if (8) fails we enlarge the class $\mathcal{N}^{\prime}$ of null sets (where $\left.F^{\prime}=F\left(S^{\prime}, \mathcal{B}^{\prime}, \mathcal{N}^{\prime}\right)\right)$ to include all sets $B^{\prime}$ on which $\phi(1)$ vanishes-i.e., in effect, we replace $S^{\prime}$ by the subspace $[\phi(1)]$.

2.2. The extension lemma. The well known process whereby the ordinary Lebesgue integral can be extended from (say) step-functions to all integrable functions can be applied here; but the details become more tricky, as infinite values give some trouble. Hence we state a general form of this process, and sketch the proof.

Let $G_{0}$ be a subset of $F$ such that

(a) $0 \in G_{0}$.

(b) If $f \in F$ and $f>0$, there exist positive real numbers $\rho_{n}$ and elements $f_{n} \geqq 0$ of $G_{0}(n=1,2, \cdots)$, such that $f=\sum \rho_{n} f_{n}$.

(c) If $f \in G_{0}$, then $f^{+}$and $-f^{-}$are in $G_{0}\left({ }^{13}\right)$.

(d) If $f \in G_{0}$ and $f \leqq 0$, then $-f \in G_{0}$.

Let $\phi_{0}$ be a mapping of $G_{0}$ in $F^{\prime}\left(=F\left(E^{\prime}\right)\right)$ such that, whenever $f, f_{1}, f_{2}, \cdots$, are in $G_{0}$,

(i) $\phi_{0}(f)>0$ if $f>0$.

(ii) $-\infty \ll \phi_{0}(f) \ll+\infty$.

(iii) If $f=\rho_{1} f_{1}+\rho_{2} f_{2}$ where $\rho_{1}, \rho_{2}$ are real (and finite), then $\phi_{0}(f)=\rho_{1} \phi_{0}\left(f_{1}\right)$ $+\rho_{2} \phi_{0}\left(f_{2}\right)$.

(iv) If $\rho_{n}$ is real and positive and $f_{n}>0(n=1,2, \cdots)$, and if $\sum \rho_{n} f_{n}$ $=f\left(\in G_{0}\right)$, then $\phi_{0}(f)=\sum \rho_{n} \phi_{0}\left(f_{n}\right)$.

(12) That is, each function $\phi\left(\chi\left(x_{n}\right)\right)$ is everywhere finite. It does not follow, in general, that the elements $x_{n}$ can be chosen so that $\phi\left(\chi\left(x_{n}\right)\right)$ is bounded.

$\left.{ }^{13}\right)$ Note that $-f^{-} \leqq 0$. In many applications, $G_{0}$ will consist of non-negative functions only, and conditions (c) and (d) are then fulfilled trivially. 
(v) $\sup \left\{\phi_{0}(f) \mid f \in G_{0}\right\} \gg 0$.

LEMмa 3. Under the above conditions, $\phi_{0}$ can be extended in one and only one way to an $F^{\prime}$-integral $\phi$ on $F$ (i.e., such that $G \supset G_{0}$ and $\phi(f)=\phi_{0}(f)$ for all $\left.f \in G_{0}\right)$.

Proof. First we note that clearly $\phi_{0}(0)=0$, and that if $f \in G_{0}$ then $-\infty \ll f$ $\ll \infty$; this follows since otherwise (c) and (d) give the existence of $g \in G_{0}$ such that $g>0$ and $g$ is somewhere infinite, and then (from (b)) there exists $h \in G_{0}$ such that $h>0$ and $g=g+h$, contradicting (ii) and (iii).

If $f \in F$ and $f>0$, we apply (b) and define $\phi_{1}(f)=\sum \rho_{n} \phi_{0}\left(f_{n}\right)$; from (iv) this agrees with $\phi_{0}$ if $f \in G_{0}$. It must, however, be shown that the definition of $\phi_{1}$ is always single-valued. Suppose, then, that $\sum \rho_{n} f_{n}=\sum \sigma_{n} g_{n}$, where $\rho_{n}, \sigma_{n}$ are real and positive and $f_{n}, g_{n}$ are non-negative elements of $G_{0}$; we must show that $\sum \rho_{n} \phi_{0}\left(f_{n}\right)=\sum \sigma_{n} \phi_{0}\left(g_{n}\right)$. One can prove that elements $h_{n m} \in F$ exist such that $h_{n m} \geqq 0, \rho_{m} f_{m}=\sum_{n} h_{m n}$, and $\sigma_{n} g_{n}=\sum_{m} h_{m n}$. From (b) we can write $h_{n m}=\sum_{p} \tau_{n m p} k_{n m p}$ where $k_{n m p} \in G_{0}, \tau_{n m p}$ is real, $k_{n m p} \geqq 0$, and $\tau_{m n p} \geqq 0$; and it follows without difficulty that $\sum \rho_{m} \phi_{0}\left(f_{m}\right)=\sum \tau_{n m p} \phi_{0}\left(k_{n m p}\right)=\sum \sigma_{n} \phi_{0}\left(g_{n}\right)$.

Clearly $\phi_{1}$ has properties $(\alpha),(\beta),(7)$, and (8) of 2.1 . Thus $\phi_{1}$ can be extended, as in 2.1 , to an $F^{\prime}$-integral $\phi$ on $F$; and it is easy to see that $\phi$ still agrees with $\phi_{0}$ on $G_{0}$. Finally, any other extension of $\phi_{0}$ to an $F^{\prime}$-integral on $F$ must agree with the $\phi$ just constructed on the successive stages of the construction, and so the extension is unique.

2.3. REMARK. Every $F^{\prime}$-integral on $F$ can be obtained by the above process with $G_{0}$ consisting of a suitable class of characteristic functions. For, using 2.1(6), we take $G_{0}$ to consist of the functions $\chi(y)$ where $y \in E$ and $y \leqq x_{n}$ for some $n ; \phi_{0}$, of course, is $\phi$ restricted to $G_{0}$. Hence an $F^{\prime}$-integral is uniquely determined by its values on the characteristic functions (as is also obvious directly).

2.4. Subspaces; induced $F^{\prime}$-integrals. Let $F=F(S, \mathcal{B}, \mathcal{N})$, let $S_{1}$ be any nonempty set in $\mathbb{B}$, and let $\mathcal{B}_{1}$ be any Borel field of subsets of $S_{1}$ such that $\mathbb{B}_{1} \subset \mathcal{B}$. Write $\mathcal{N}_{1}=\mathcal{N} \cap B_{1}$; we shall say that the function space $F_{1}=F\left(S_{1}, B_{1}, \mathcal{N}_{1}\right)$ is a subspace of $F$. This amounts to defining $F\left(E_{1}\right)$ to be a "subspace" of $F(E)$ if $E_{1}$ is a subalgebra of a principal ideal in $E$. We can regard the subspace $F_{1}$ as imbedded in $F$ in a natural way, each function $f \in F_{1}$ corresponding to the function in $F$ which agrees with $f$ on $S_{1}$ and is 0 on $S-S_{1}$.

Suppose now that $\phi$ is an $F^{\prime}$-integral on $F$, with $G$ as its set of integrable functions, and that $F_{1}$ is a subspace of $F$ (imbedded in $F$ as above). Define $G_{1}=F_{1} \cap G$, and let $\bar{\phi}$ denote the mapping $\phi$ restricted to $G_{1}$. It is easy to verify that $\Phi$ and $G_{1}$ satisfy all but (6) (or $\beta$ ) and (8) of the requirements (2.1) for an $F^{\prime}$-integral on $F_{1}$. If $\Phi$ also satisfies $2.1(6)$ and (8), we say that $\Phi$ is the $F^{\prime}$-integral on $F_{1}$ induced by $\phi$.

2.5. Isomorphism and refinement of integrals. Let $\phi$ be an $F^{\prime}$-integral on $F$, and let $\psi, \psi^{\prime}$ be isomorphisms of $F, F^{\prime}$, respectively, onto function spaces 
$F_{1}, F_{1}^{\prime}$. Then, defining $\bar{\phi}=\psi^{\prime} \phi \psi^{-1}$, we clearly have that $\Phi$ is an $F_{1}^{\prime}$-integral on $F_{1}$; we say that $\phi$ is isomorphic to $\phi$. If the isomorphism $\psi$ is strict and $\psi^{\prime}$ is the identity, we say that $\phi$ is strictly isomorphic to $\phi$; thus in this case we have $\Phi=\phi \psi^{-1}$, where $\psi$ is induced by an isomorphism of $E$.

Another notion which will arise below is that of a refinement of an $F^{\prime}$-integral. Suppose that $\phi$ is an $F^{\prime}$-integral on $F$, and that $\phi^{\prime}$ is an $F^{\prime \prime}$-integral on the same function space $F$, where $F^{\prime \prime}$ is another function space. Let $\phi$ and $\phi^{\prime}$ have $G$ and $G^{\prime}$ as their respective sets of integrable functions. If $G \subset G^{\prime}$, and if, for $f, g \in G$, we have $\phi^{\prime}(f)=\phi^{\prime}(g)$ if and only if $\phi(f)=\phi(g)$, we call $\phi^{\prime}$ a refinement of $\phi$. It will in general happen (see 7.9) that more functions are "integrable $\phi$ " than are "integrable $\phi . "$

\section{Function-valued MEASURES AND INTEgRals}

3.1. Given an $F^{\prime}$-integral $\phi$ on $F=F(E)$, we define, for each $x \in E$,

$$
\lambda(x)=\phi(\chi(x)) .
$$

Then $\lambda$ can be thought of as a generalized "measure" on $E$. It is easy to verify that $\lambda$ has the properties:

(2) $\lambda(o)=0$, and if $x \in E$ and $x \neq 0$ then $\lambda(x)>0$.

(3) If $x_{n} \in E$ and $x_{m} x_{n}=o(m, n=1,2, \cdots, m \neq n), \lambda\left(\bigvee x_{n}\right)=\sum \lambda\left(x_{n}\right)$.

(4) $e=V x_{n}$ where $\lambda\left(x_{n}\right) \ll \infty(n=1,2, \cdots)\left({ }^{14}\right)$.

(5) $\lambda(e) \gg 0$.

Quite generally, any mapping $\lambda$ of $E$ in $F^{\prime}=F\left(E^{\prime}\right)$, where $E$ and $E^{\prime}$ are Boolean $\sigma$-algebras satisfying the countable chain condition $\left({ }^{15}\right)$, having properties (2)-(5), will be called an $F^{\prime}$-measure on $E$, and the pair $(E, \lambda)$ will be called an $F^{\prime}$-measure algebra. Thus every $F^{\prime}$-integral $\phi$ on $F(E)$ "induces" (by (1)) an $F^{\prime}$-measure $\lambda$ on $E$. Conversely, as follows easily from Lemma 3 (2.2) and the remark in 2.3, every $F^{\prime}$-measure $\lambda$ on $E$ determines a unique $F^{\prime}$-integral $\phi$ on $F(E)$ which induces $\lambda$. The construction of $\phi$ from $\lambda$ is analogous to the process of integration; in fact, if $f \in F$ and if (for simplicity) $f \geqq 0$ and $[f] \leqq x_{r}$ for some $r$ (cf. 2.1(6)), then (using any realization of $F$ as $F(S, \mathcal{B}, \mathcal{N})$ ) we define $x_{k n}=$ element of $E$ corresponding to $\{s \mid k / n<f(s)$ $\leqq(k+1) / n\}$ and $x_{\infty}=$ element of $E$ corresponding to $\{s \mid f(s)=\infty\}$, and readily show that as $n \rightarrow \infty$ the sum $\sum_{k=0}^{\infty}(k / n) \lambda\left(x_{k n}\right)+\infty \lambda\left(x_{\infty}\right)$ converges to $\phi(f)$. Accordingly we shall of ten write $\phi(f)$ as $\int f d \lambda(f \in G \subset F)$.

3.2. Three examples of this process will be of particular importance in the sequel. One is the case in which $E^{\prime}$ reduces to a single atom. Here the function space $F^{\prime}$ reduces to the set of real numbers, extended by $\pm \infty$; the $F^{\prime}$-measure algebras in this case coincide with the ordinary $\sigma$-finite numerical measure algebras, and the corresponding $F^{\prime}$-integrals are the usual Lebesgue

(14) It does not follow that we can take $\lambda\left(x_{n}\right)$ to be bounded; cf. footnote 12.

(15) It suffices to assume only that $E^{\prime}$ satisfies the countable chain condition; that $E$ must also satisfy this condition then follows from the conditions on $\lambda$. 
integrals with respect to the numerical measures. Throughout this paper, the term "numerical measure algebra" will be used for this case, i.e., for a $\sigma$-finite numerical measure algebra.

The opposite extreme is provided by the case in which $\phi$ is an isomorphism of $F$ onto $F^{\prime}$. We then have, from $1.6, \lambda(x)=f_{0} \chi(\theta(x))$ for all $x \in E$, where $\theta$ is an isomorphism of $E$ onto $E^{\prime}$ and $f_{0}$ is a fixed function in $F^{\prime}$, everywhere positive and finite $\left({ }^{16}\right)$.

A more typical example (as will be shown later) is the "direct product" of these two types, of the form $(J, m) \times E^{\prime}$ where $(J, m)$ is an arbitrary $\left(\sigma\right.$-finite) measure algebra $\left({ }^{17}\right)$. For its definition and properties we refer to $[5, \S 4]$, where it is shown that this product can be realized in the product $R \times S^{\prime}$ of the representation spaces of $J$ and $E^{\prime}$ respectively, and has a particular $F^{\prime}$-measure $M$ (where $F^{\prime}=F\left(E^{\prime}\right)$ ), realized by continuous functions on $S^{\prime}$ modulo sets of first category, as in (1.9) ${ }^{(18)}$. The fundamental property of $M$ is that, for every "measurable" subset $H$ of $R \times S^{\prime}$, the value of $M(H)$ at $s \in S^{\prime}$ is (except for a first category set of $s^{\prime} s$ ) the $m$-measure of the section of $H$ over $s$. We shall refer to this $M$ as the "standard" $F^{\prime}$-measure on $(J, m)$ $\times E^{\prime}$, and to the corresponding $F^{\prime}$-integral $\phi$, where $\phi(f)=\int f d M$, as the "standard" $F^{\prime}$-integral on $F\left((J, m) \times E^{\prime}\right)$. We have:

THEOREM 2. The standard integral $\int f d M$ on $F\left((J, m) \times E^{\prime}\right)$ is the function on $S^{\prime}$ whose value at each $s \in S^{\prime}$, except for a set of first category, is $\int_{R} f(x, s) d m(x)$.

(The last integral here is, of course, an ordinary numerical one.)

It is sufficient to prove this when $f \geqq 0$ and $[f] \leqq x_{r}$; but then the construction indicated in 3.1 gives for each $s \in S^{\prime}$, if we exclude at most countably many exceptional sets of first category, a corresponding (numerical) Lebesgue sum for the function $f(x, s)$ and the measure $m$.

3.3. For any $F^{\prime}$-measure algebra $(E, \lambda)$ we write (in accordance with the notation in [5]) $x \sim y$ (where $x, y \in E)$ to mean $\lambda(x)=\lambda(y)$. The system $(E, \lambda)$ then satisfies two of the postulates of [5], viz.

(0) $E$ is a Boolean $\sigma$-algebra satisfying the countable chain condition, and $\sim$ is an equivalence relation on $E$.

(I) If $x=\mathrm{V} x_{n}, y=\mathrm{V} y_{n}$, where $x_{m} x_{n}=o=y_{m} y_{n}(m \neq n, m, n=1,2, \cdots)$, and if $x_{n} \sim y_{n}$ for all $n$, then $x \sim y$.

Any system $(E, \sim)$ satisfying these postulates and the following two further ones:

(16) This is essentially the "trivial" case of [5, p. 283].

$\left.{ }^{17}\right)$ Throughout what follows, all numerical measure algebras referred to will be assumed to be $\sigma$-finite. In [5], $(J, m)$ was assumed in addition to satisfy postulates II and III of 3.3 below; but these are not needed for the construction of $(J, m) \times E^{\prime}$.

${ }^{(18)}$ The representation space realizations $R, S^{\prime}$ of $J, E^{\prime}$ are convenient, but any other realizations would do; the values of $M$ would in general be equivalence classes of "measurable" functions on $S^{\prime}$, modulo "null" sets. 
(II) Given $x^{\prime} \sim x \geqq y$, there exists $y^{\prime} \leqq x^{\prime}$ such that $y^{\prime} \sim y$;

(III) If $x \sim y$, there exist bounded( $\left.{ }^{19}\right)$ elements $x_{n}, y_{n}(n=1,2, \cdots)$ such that $x=\mathrm{V} x_{n}, y=\mathrm{V} y_{n}, x_{m} x_{n}=o=y_{m} y_{n}(m \neq n)$, and $x_{n} \sim y_{n}$;

is an "abstract measure algebra" in the sense of [5]. It was proved in $[5, \S 19]$ that every abstract measure algebra arises from a suitable $F^{\prime}$-measure algebra. The converse is false; even a numerical measure algebra need not satisfy II or III, if it is allowed to be atomic. We shall, however, prove below (Theorem $6,5.1$ ) that every $F^{\prime}$-measure algebra can be imbedded in one which does satisfy II and III (and some further conditions as well), and which therefore is an abstract measure algebra.

3.4. Two $F^{\prime}$-measure algebras $\left(E_{1}, \lambda_{1}\right),\left(E_{2}, \lambda_{2}\right)$ are said to be isomorphic if there is an algebraic $\sigma$-isomorphism $\theta$ of $E_{1}$ onto $E_{2}$ which preserves the relation $\sim$ introduced above-i.e., for which $\lambda_{2}(\theta(x))=\lambda_{2}(\theta(y))$ if and only if $\lambda_{1}(x)=\lambda_{1}(y)$. (This agrees with the use of the term "isomorphism" in [5].) If further $\theta$ can be chosen so that there is an isomorphism $\psi$ of $F^{\prime}$ onto itself such that $\lambda_{2}(\theta(x))=\psi\left(\lambda_{1}(x)\right)$ (for all $x \in E$ ), then $\left(E_{1}, \lambda_{1}\right)$ and $\left(E_{2}, \lambda_{2}\right)$ will be called " $F^{\prime}$-isomorphic"; and they will be called "strictly $F^{\prime}$-isomorphic" if $\psi$ here can be taken to be the identity, i.e., if $\lambda_{2}(\theta(x))=\lambda_{1}(x)$.

\section{Full-VAlued $F^{\prime}$-MEASURES}

4.1. In this section we shall discuss a class of $F^{\prime}$-measure algebras which will be a useful tool in $\S 5$, incidentally showing (Theorems 3 and 4 below) that this class is substantially equivalent to the "abstract measure algebras" mentioned in 3.3.

Definition. An $F^{\prime}$-measure algebra $(E, \lambda)$ will be called "full-valued" if it satisfies:

(1) Given $x \in E$ and $f^{\prime} \in F^{\prime}$ such that $0 \leqq f^{\prime} \leqq \lambda(x)$, there exists $y \in E$ such that $y \leqq x$ and $\lambda(y)=f^{\prime}$.

Clearly full-valuedness is invariant under $F^{\prime}$-isomorphism. A numerical measure algebra is full-valued if and only if it is nonatomic. It is easily seen from (1) that:

(2) If $(E, \lambda)$ is full-valued, a necessary and sufficient condition that $x \in E$ be bounded is that $\lambda(x)$ be finite (i.e., $\lambda(x) \ll \infty)$.

TheOREM 3. Every full-valued $F^{\prime}$-measure algebra $(E, \lambda)$ satisfies the postulates (cf. 3.3) for an abstract measure algebra; further, $(E, \lambda)$ has no nonzero indecomposable elements $\left({ }^{20}\right)$, and its invariant elements $\left({ }^{21}\right)$ form a subalgebra $U$ of $E$ which is isomorphic to $E^{\prime}$, where $F^{\prime}=F\left(E^{\prime}\right)$.

(19) An element $x \in E$ is "bounded" if the relations $x \sim y$ and $y \leqq x$ together imply $y=x$.

(20) An element $x \in E$ is "indecomposable" (with respect to $\lambda$ ) if the relations $y \leqq x, z \leqq x$, $y z=o$ and $\lambda(y)=\lambda(z)$ together imply $y=z=o$.

(21) An element $u \in E$ is "invariant" in $(E, \lambda)$ if the relations $y \leqq u$ and $\lambda(y)=\lambda(z)$ together imply $z \leqq u$. 
Postulate II (3.3) follows trivially from (1), and III then follows, in view of (2), by the same argument as in [5, p. 289]. That the only indecomposable element is $o$ is also trivial from (1). The invariant elements are known [5, p. 290] to form a $(\sigma-)$ subalgebra $U$ of $E$. We map $U$ in $E^{\prime}$ by assigning to $u \in U$ the element $[\lambda(u)] \in E^{\prime}$. It is easy to verify that (for any elements $\left.x_{n}, x \in E\right)\left[\lambda\left(\bigvee x_{n}\right)\right]=\mathrm{V}\left[\lambda\left(x_{n}\right)\right],[\lambda(x)]=o^{\prime}$ if and only if $x=o$, and $[\lambda(e)]=e^{\prime}$, where $o^{\prime}, e^{\prime}$ denote the zero and unit elements of $E^{\prime}$. If further $u_{1}, u_{2} \in U$ and $u_{1} u_{2}=o$, then $\left[\lambda\left(u_{1}\right)\right]\left[\lambda\left(u_{2}\right)\right]=o^{\prime} ;$ for if not, the function $f^{\prime}=\inf \left\{\lambda\left(u_{1}\right), \lambda\left(u_{2}\right)\right\}$ is positive, and there exist $y_{1} \leqq u_{1}$ and $y_{2} \leqq u_{2}$ such that $\lambda\left(y_{1}\right)=f^{\prime}=\lambda\left(y_{2}\right)$, contradicting the invariance of $u_{1}$. It readily follows that the mapping $u \rightarrow[\lambda(u)]$ is a $(\sigma-)$ isomorphism of $U$ onto some subalgebra of $E^{\prime}$. Finally, given $x^{\prime} \in E^{\prime}$, there exists $y \leqq e$ such that $\lambda(y)=\lambda(e) \chi\left(x^{\prime}\right)$; the greatest such $y$ is easily seen to be invariant, and (because $\lambda(e) \gg 0)[\lambda(y)]=x^{\prime}$. Thus the mapping is onto $E^{\prime}$.

REMARK. The converse of Theorem 3 is also true; every abstract measure algebra $(E, \sim)$ without indecomposable elements (other than $o$ ) is isomorphic to a full-valued $F^{\prime}$-measure algebra, where $F^{\prime}=F(U), U$ being the algebra of invariant elements of $(E, \sim)$. For, from $[5, \S 19],(E, \sim)$ is isomorphic to a principal ideal in a direct product $(J, m) \times U$, where $J$ is nonatomic; now this direct product is full-valued [5, pp. 288, 289], and therefore so is the principal ideal.

\subsection{THEOREM 4. Every isomorphism between two full-valued $F^{\prime}$-measure} algebras is an $F^{\prime}$-isomorphism (cf. 3.4).

Let $\left(E_{1}, \lambda_{1}\right)$ and $\left(E_{2}, \lambda_{2}\right)$ be isomorphic full-valued $F^{\prime}$-measure algebras, where $F^{\prime}=F\left(E^{\prime}\right)$. There is no loss of generality in supposing that the isomorphism $\theta$ between $E_{1}$ and $E_{2}$ is the identity; thus we take $E_{1}=E_{2}=E$, and have that $\lambda_{1}$ and $\lambda_{2}$ induce the same relation $\sim$ on $E$, and so give rise to the same subalgebra $U$ of invariant elements of $E$. As the proof of Theorem 3 shows, we may (after carrying out an isomorphism on $E^{\prime}$ and so on $F^{\prime}$ ) take $E^{\prime}=U$ and $\left[\lambda_{1}(u)\right]=u$ whenever $u \in U$. Similarly, after a (different) isomorphism of $F^{\prime}$ we can take $\left[\lambda_{2}(u)\right]=u(u \in U)$. It will suffice to show (see 1.6, Corollary 1) that, assuming both these relations simultaneously, there exists $f_{0} \in F^{\prime}$ such that $0 \ll f_{0} \ll \infty$ and $\lambda_{1}(x)=f_{0} \lambda_{2}(x)$ for all $x \in E$. For convenience of description, we realize $F^{\prime}$ as $F\left(S^{\prime}, \mathcal{B}^{\prime}, \mathcal{N}^{\prime}\right)$, and do not distinguish between elements of $E^{\prime}(=U)$ and the corresponding subsets of $S^{\prime}$.

We first note

$$
\left.\lambda_{1}(x u)=\lambda_{1}(x) \chi(u) \quad \text { (for all } x \in E \text { and } u \in U\right),
$$

with a similar relation for $\lambda_{2}$. For $\lambda_{1}(x u) \leqq \inf \left\{\lambda_{1}(x), \lambda_{1}(u)\right\}$; now we are assuming $\left[\lambda_{1}(u)\right]=u$, so $\lambda_{1}(u) \leqq \infty \chi(u)$, giving $\lambda_{1}(x u) \leqq$ inf $\left\{\lambda_{1}(x), \infty \chi(u)\right\}$ $=\lambda_{1}(x) \chi(u)$. Similarly $\lambda_{1}(x-x u) \leqq \lambda_{1}(x) \chi(-u)$; and addition shows that the last two inequalities must both be equalities. 
In view of Theorem 3 , we can apply $[5, \S 10]$ to $(E, \sim)$ to obtain (with a slight change of notation) $e=e^{1} \vee e^{\prime \prime}$, where $e^{1}$ and $e^{\prime \prime}$ are disjoint and invariant (and so in $E^{\prime}$ ), and $e^{1}$ is the greatest bounded invariant element of $(E, \sim)$. From (2) and (3), $e^{1}$ is the subset of $S^{\prime}$ on which $\lambda_{1}(e)$ is finite; and the same will apply to $\lambda_{2}$. Thus, from (3) again, $\lambda_{1}\left(e^{1}\right)$ and $\lambda_{2}\left(e^{1}\right)$ are positive and finite at each $s^{\prime} \in e^{1}$ (except for "null" sets), so that there exists $g^{\prime} \in F^{\prime}$ such that $0 \ll g^{\prime} \ll \infty$ on $e^{1}$ (i.e., $0<g^{\prime}\left(s^{\prime}\right)<\infty$ for each $s^{\prime} \in e^{1}-N^{\prime}$ where $\left.N^{\prime} \in \mathcal{N}^{\prime}\right), g^{\prime}=0$ outside $e^{1}$, and

$$
\lambda_{1}\left(e^{1}\right)=g^{\prime} \lambda_{2}\left(e^{1}\right)
$$

We shall deduce:

$$
\lambda_{1}(x)=g^{\prime} \lambda_{2}(x) \quad \text { whenever } x \in E\left(e^{1}\right)\left({ }^{22}\right) .
$$

For suppose this false; then there is no loss of generality in assuming that $\lambda_{1}(x)>g^{\prime} \lambda_{2}(x)$ for a set of $s^{\prime \prime}$ s not in $\mathcal{N}^{\prime}$, and hence that there exist $u \in E^{\prime}$ and integers $k, n$ such that $u \neq o^{\prime}, 1 \leqq k \leqq n$, and the relations $\lambda_{1}(x)$ $\geqq(k / n) \lambda_{2}\left(e^{1}\right),((k-1) / n) \lambda_{2}\left(e^{1}\right) \geqq \lambda_{2}(x)$ hold for all $s^{\prime} \in u$. That is, from (3), $\lambda_{1}(x u) \geqq(k / n) \lambda_{1}(u)$ and $((k-1) / n) \lambda_{2}(u) \geqq \lambda_{2}(x u)$. But since $\lambda_{1}$ is full-valued we readily construct $n$ disjoint elements $x_{1}, x_{2}, \cdots, x_{n} \in E$ such that $\bigvee x_{i}=u, \lambda_{1}\left(x_{i}\right)=(1 / n) \lambda_{1}(u)$, and $x_{1} \bigvee \cdots \vee x_{k}=x u$. Since $x_{1} \sim x_{2} \sim \cdots \sim x_{n}$, we have $\lambda_{2}\left(x_{i}\right)=(1 / n) \lambda_{2}(u)$, and therefore $\lambda_{2}(x u) \geqq(k / n) \lambda_{2}(u)$, giving a contradiction.

Next we consider $E\left(e^{\prime \prime}\right)$. From $[5, \S 10]$ we have $e^{\prime \prime}=\mathrm{V} f^{i}(i=1,2, \cdots$, to $\infty)$, where the elements $f^{i}$ are bounded and disjoint and $f^{1} \sim f^{2} \sim \cdots \sim f^{i}$ $\sim \ldots$. Thus we have $\lambda_{1}\left(f^{1}\right)=\lambda_{1}\left(f^{i}\right)$ and $\lambda_{2}\left(f^{1}\right)=\lambda_{2}\left(f^{i}\right), 0 \ll \lambda_{1}\left(f^{1}\right) \ll \infty$ on $e^{\prime \prime}$, and $0 \ll \lambda_{2}\left(f^{1}\right) \ll \infty$ on $e^{\prime \prime}$. Defining $g^{\prime \prime} \in F^{\prime}$ so that $\lambda_{1}\left(f^{1}\right)=g^{\prime \prime} \lambda_{2}\left(f^{1}\right)$ and $g^{\prime \prime}=0$ outside $e^{\prime \prime}$, we have $0 \ll g^{\prime \prime} \ll \infty$ on $e^{\prime \prime}$, and the same argument as before (but with $x_{1} \bigvee x_{2} \bigvee \cdots \bigvee x_{n}=f^{i} u$ instead of $u$ ) shows that $\lambda_{1}(x)=g^{\prime \prime} \lambda_{2}(x)$ whenever $x \leqq f^{i}$, and hence also whenever $x \leqq e^{\prime \prime}$.

Finally, let $f_{0}=g^{\prime}+g^{\prime \prime}$; thus $f_{0} \in F$, and the preceding results combine to give $0 \ll f_{\theta} \ll \infty$ and $\lambda_{1}(x)=f_{0} \lambda_{2}(x)$ for all $x \in E$.

4.3. Theorem 5. Every full-valued $F^{\prime}$-measure algebra is strictly $F^{\prime}$-isomorphic to a principal ideal in a direct product $(J, m) \times E^{\prime}$ (with standard $F^{\prime}$-measure), where $(J, m)$ is a nonatomic numerical measure algebra and $F^{\prime}=F\left(E^{\prime}\right)$.

(It will follow that if two full-valued $F^{\prime}$-measure algebras are isomorphic, they are strictly $F^{\prime}$-isomorphic, but in general under a different isomorphism.)

For, in view of Theorem $3,[5, \S 19]$ shows that an $F^{\prime}$-measure algebra $(E, \lambda)$ is isomorphic to such a principal ideal; and, from Theorem 4 , this isomorphism must be an $F^{\prime}$-isomorphism. It can be replaced by a strict $F^{\prime}$-iso-

${ }^{(22)}$ The notation $E(x)$ denotes the principal ideal of $x$ in $E$; that is, the set of all $y \in E$ such that $y \leqq x$. 
morphism (see 3.4) as follows. We have each $x \in E$ represented by an equivalence class of "measurable" subsets, say $H$, where each $H$ is a subset of a fixed set $K$ in $R \times S^{\prime}$ (cf. 3.2); here $S^{\prime}$ is (say) the representation space of $E^{\prime}$, and $R$ can be any realization of $(J, m)$. This representation is an isomorphism and we have $\lambda(x)=f_{0} M(H), M$ denoting the "standard" measure function on the product and $f_{0}$ being a fixed (continuous) function on $S^{\prime}$ such that $0 \ll f_{0} \ll \infty$. Now it follows from [4] (or from the construction in [5]) that, after enlarging $(J, m)$ if necessary, $(J, m)$ can be realized by the (ordinary) direct product of a measure space $(W, \mu)$ with the real line $R_{1}$ in its usual measure, and so we may suppose that $R$ is the usual direct product of $R_{1}$ and $W$. Let $\theta$ be the mapping of $R \times S^{\prime}=R_{1} \times W \times S^{\prime}$ in itself which sends the point $\left(r_{1}, w, s^{\prime}\right)$ into $\left(r_{1} f_{0}\left(s^{\prime}\right), w, s^{\prime}\right)$. It is not hard to verify that $\theta$ maps "measurable" sets (in $R \times S^{\prime}$ ) onto "measurable" sets, and "null" sets onto "null" sets, provided we "complete" the class of "measurable" (i.e., restricted Borel) sets, in the description given in $[5, \S 4]$, by adjoining all subsets of "null" sets. Clearly $\lambda(x)=M(\theta(H))$, and we have only to take the new representative of $x$ to be the equivalence class of the set $\theta(H)$.

Remark. Conversely, every such principal ideal in $(J, m) \times E^{\prime}$, where $J$ is nonatomic, is full-valued (cf. the remark at the end of 4.1); thus Theorem 5 characterizes full-valued $F^{\prime}$-measure algebras.

Note that, in the isomorphism of Theorem 5, the invariant algebra $U$ of $E$ is mapped isomorphically onto the algebra of "cylinder sets," of the form $K \cap\left(R \times u^{*}\right)$ where $u^{*}$ is a "measurable" subset of $S^{\prime}$. For these sets (modulo "null" sets) give precisely the invariant elements of the principal ideal corresponding to $K$ in $(J, m) \times E^{\prime}$.

\section{IMBEDDING OF $F^{\prime}$-MEASURES AND INTEGRALS}

5.1. We have just seen that the theory of full-valued $F^{\prime}$-measure algebras is essentially the same as that of abstract measure algebras. The next theorem shows that this theory suffices to cover the general $F^{\prime}$-measure algebras.

Theorem 6. Given an $F^{\prime}$-measure algebra $(E, \lambda)$, where $F^{\prime}=F\left(E^{\prime}\right)$, there exists a full-valued $F^{\prime}$-measure algebra $\left(E^{*}, \lambda^{*}\right)$ such that $(E, \lambda)$ is strictly $F^{\prime}$-isomorphic to a subalgebra of $\left(E^{*}, \lambda^{*}\right)$; more precisely, $E$ is a subalgebra of $E^{*}$ and $\lambda^{*}$ agrees with $\lambda$ on $E$.

Proof. The first step is to imbed $(E, \lambda)$ in an $F^{\prime}$-measure algebra $(P, L)$ with the further property:

(1) Given $x \in P$ and a real number $\sigma$ such that $0 \leqq \sigma \leqq 1$, there exists $y \in P$ such that $y \leqq x$ and $L(y)=\sigma L(x)$.

To do this, let $(I, \mu)$ denote the measure algebra of the (Lebesgue) measurable sets modulo null sets on the unit interval, and form the direct product $\left(P, M_{1}\right)=(I, \mu) \times E$ (cf. 3.2), the factor $E$ being taken "trivial" here. Then 
$\left(P, M_{1}\right)$ is a full-valued $F$-measure algebra. For each $x \in P$, define $L(x)$ $=\int M_{1}(x) d \lambda$ (cf. 3.1); it is easy to see that $(P, L)$ is an $F^{\prime}$-measure algebra, and (1) follows from the full-valuedness of $M_{1}$. Further, the elements of $P$ corresponding to "cylinder sets" $I \times x, x \in E$, form a sub-algebra of $P$ isomorphic to $E$, and clearly (from 3.1(1)) $L(I \times x)=\lambda(x)$ if $x \in E$. Thus, after subjecting $P$ to an isomorphism, we may identify $E$ with this subalgebra of $P$, and have that $L$ agrees with $\lambda$ on $E$.

Next we form a "direct product" $(P, L) \times E^{\prime}$, as follows $\left({ }^{23}\right)$. Consider the direct product $R \times S^{\prime}$ of the representation spaces of $P$ and $E^{\prime}$ respectively; the choice of representation space realizations is not here purely for convenience, and we shall make essential use of the compactness of $R$ later. As in 1.9 we realize $F^{\prime}$ as the family of continuous functions on $S^{\prime}$, modulo sets of first category in $S^{\prime}$. Any subset of $R \times S^{\prime}$ of the form $q^{*} \times x^{\prime *}$, where $q^{*}$ is a (possibly empty) open-closed subset of $R$ corresponding to $q \in P$, and similarly $x^{\prime *}$ is open-closed in $S^{\prime}$ and corresponds to $x^{\prime} \in E^{\prime}$, will be called a "rectangle." For each rectangle $r=q^{*} \times x^{\prime *}$, write $L(q) \chi\left(x^{\prime *}\right)=\lambda_{1}(r)$; our object will be to extend $\lambda_{1}$ to the required "measure."

Let $R_{0}$ be a fixed open-closed subset of $R$ corresponding to an element $q_{0} \in P$ for which $L\left(q_{0}\right) \ll \infty$. For any set $H \subset R_{0} \times S^{\prime}$, we call a sequence $\left\{r_{n}\right\}$ of rectangles "admissible" for $H$ if $\bigcup r_{n} \cup\left(R \times N^{\prime}\right) \supset H$, where $N^{\prime}$ is some set of first category in $S^{\prime}$. Define

$$
\lambda_{0}^{*}(H)=\inf \sum_{n=0}^{\infty} \lambda_{1}\left(r_{n}\right),
$$

the infimum being taken over all sequences $\left\{r_{n}\right\}$ which are admissible for $H$; since $F^{\prime}$ satisfies the countable chain condition, this infimum exists in $F^{\prime}$. We shall show:

(3) Given $H \subset R_{0} \times S^{\prime}$ and $\epsilon>0$, there exists an admissible sequence $\left\{r_{n}\right\}$ such that $\sum \lambda_{1}\left(r_{n}\right) \leqq \lambda_{0}^{*}(H)+\epsilon$.

For, from the countable chain condition, there exists a sequence of admissible sequences, say $\left\{r_{n i}\right\}$, such that $\inf _{i} \sum_{n} \lambda_{1}\left(r_{n i}\right)=\lambda_{0}{ }^{*}(H)$ and $H$ $\subset \bigcup_{n} r_{n i} \cup\left(R_{0} \times N_{i}^{\prime}\right)$, where $N_{i}^{\prime}$ is of first category in $S^{\prime}$. Since $\lambda_{1}$ is non-negative and finitely additive for rectangles, we can further suppose that the rectangles $r_{n i}$ are, for fixed $i$, disjoint, that $r_{n i} \subset R_{0} \times S^{\prime}$, and that each $r_{n, i+1}$ is contained in some $r_{m i}$. Let $f_{i}^{\prime}=\sum_{n} \lambda_{1}\left(r_{n i}\right)$; then $0 \leqq f_{i}^{\prime} \ll \infty, f_{1}^{\prime} \geqq f_{2}^{\prime}$ $\geqq \cdots$, and $\lim f_{i}^{\prime}=\lambda_{0}^{*}(H)$. By an "Egoroff argument" (cf. [5, p. 286]) there exist disjoint open-closed sets $x_{\mathbf{k}}^{\prime *}$ in $S^{\prime}(k=1,2, \cdots)$ such that (i) $U x_{\mathbf{k}}^{\prime *}=S^{\prime}-N^{\prime}$ where $N^{\prime}$ is of first category, (ii) to each $k$ corresponds an $i(k)$ such that $f_{i(\boldsymbol{k})}^{\prime}-\lambda_{0}^{*}(H)<\epsilon$ at each point of $x_{\mathbf{k}}^{\prime *}$. Consider the rectangles $R_{n k}$

${ }^{(23)}$ One can define, using similar ideas, a "direct product" of any two $F^{\prime}$-measure algebras (under slight restrictions); but at this generality few useful properties of the direct product survive. Even in the present case it will be seen that if $E^{\prime}$ is isomorphic to $P$, the "direct product" $(P, L) \times E^{\prime}$ is concentrated on the diagonal, and is strictly $F^{\prime}$-isomorphic to $(P, L)$. 
$=\left(R \times x_{k}^{\prime *}\right) \cap r_{n, i(k)}$; we evidently have $\bigcup_{n, k} R_{n k} \cup\left\{R \times\left(N^{\prime} \cup \cup N_{i}^{\prime}\right)\right\} \supset H$ and $\sum_{n, k} \lambda_{1}\left(R_{n k}\right)-\lambda_{0}{ }^{*}(H) \leqq \epsilon$ everywhere. On renumbering $\left\{R_{n k}\right\}$ into a single sequence, we obtain an admissible sequence establishing (3).

We shall deduce:

(4) If $H_{m} \subset R_{0} \times S^{\prime}(m=1,2, \cdots)$, then $\lambda_{0}{ }^{*}\left(\cup H_{m}\right) \leqq \sum \lambda_{0}{ }^{*}\left(H_{m}\right)$.

For, from (3), there is an admissible sequence $\left\{r_{m n}\right\}$ for $H_{m}$ such that $\sum_{n} \lambda_{1}\left(r_{m n}\right) \leqq \lambda^{*}\left(H_{m}\right)+\epsilon / 2^{m}$; and the double sequence $\left\{r_{m n}\right\}$ is admissible for $U H_{m}$.

Thus $\lambda_{0}{ }^{*}$ is an "outer $F^{\prime}$-measure" on $R_{0} \times S^{\prime}$, in the sense that $\lambda_{0}^{*}(0)=0$, $\lambda_{0}^{*}(H) \leqq \lambda_{0}^{*}(K)$ if $H \subset K$, and (4) holds. The Carathéodory theory of outer measure (see e.g. [9, Chap. II]) applies here almost literally unchanged to show that $\lambda_{0}{ }^{*}$ is countably additive on a Borel field of "measurable" subsets of $R_{0} \times S^{\prime}$. It is easy to see that every rectangle $r$ is "measurable"-i.e., that if $H \subset r$ and $K C\left(R_{0} \times S^{\prime}\right)-r$ then $\lambda_{0}^{*}(H \cup K)=\lambda_{0}{ }^{*}(H)+\lambda_{0}^{*}(K)$. Further,

(5) $\lambda_{0}^{*}(r)=\lambda_{1}(r)$ if $r$ is a rectangle.

For suppose $r=q^{*} \times x^{*}$, and let $\left\{r_{n}\right\}$ be an admissible sequence, covering $r-\left(R \times N^{\prime}\right)$ where $N^{\prime}$ is of first category. Let $r_{n}=q_{n}{ }^{*} \times x_{n}^{\prime *}$, and consider any $s_{0}^{\prime} \in S^{\prime}-N^{\prime}$. Since the "section" $q^{*} \times s_{0}^{\prime}$ is compact and the rectangles $r_{n}$ are open, a finite number of them cover $q^{*} \times s_{0}^{\prime}$; say $q^{*} \subset q_{n_{1}}^{*} \cup \cdots \cup q_{n_{\nu}}^{*}$, where $s_{0}^{\prime} \in x_{n_{1}}^{\prime *} \cap \cdots \cap x_{n_{\nu}}^{\prime *}$. Then $L(q) \leqq L\left(q_{n_{1}}\right)+\cdots+L\left(q_{n_{\nu}}\right)$, a relation which holds for all $s^{\prime} \in S^{\prime}$ (and not merely at each $s^{\prime}$ in a residual set, as would be the case if we were dealing with infinite sums) if we suppose, as we may, that these functions are everywhere continuous; hence, since $\chi\left(x^{\prime *}\right), \chi\left(x_{n_{1}}^{\prime *}\right), \cdots, \chi\left(x_{n_{\nu}}^{\prime *}\right)$ all have the value 1 at $s_{0}^{\prime}$, we have $\lambda_{1}(r) \leqq \lambda_{1}\left(q_{n_{1}}\right)+\cdots+\lambda_{1}\left(q_{n_{v}}\right)$ at $s_{0}^{\prime}$. Again, for all $s^{\prime} \in S^{\prime}$ the value of the continuous function denoted by $\sum_{n=0}^{\infty} \lambda_{1}\left(q_{n}\right)$ (cf. 1.9) is at least as great as that of $\lambda_{1}\left(q_{n_{1}}\right)+\cdots+\lambda_{1}\left(q_{n_{p}}\right)$. Thus $\lambda_{1}(r)$ $\leqq \sum_{n=0}^{\infty} \lambda_{1}\left(q_{n}\right)$ at $s_{0}^{\prime}$, for all $s_{0}^{\prime} \in S^{\prime}-N^{\prime}$. This proves $\lambda_{1}(r) \leqq \lambda_{0}^{*}(r)$, and the reverse inequality is trivial.

Now, from 3.1(4), there exists a sequence of disjoint open-closed subsets $R_{n}$ of $R(n=1,2, \cdots)$ such that $R-\bigcup R_{n}$ is of first category in $R$, and each $R_{n}$ corresponds to an element $q_{n}$ of $P$ such that $L\left(q_{n}\right) \ll \infty$. Let $\lambda_{n}^{*}$ be the $F^{\prime}$-measure on $R_{n} \times S^{\prime}$ defined by the above process, $R_{0}$ being replaced by $R_{n}$. For every $H \subset R \times S^{\prime}$ we define

$$
\lambda^{*}(H) \cap \sum \lambda_{n}^{*}\left(H \cap\left(R_{n} \times S^{\prime}\right)\right),
$$

and we define the field $B$ of "measurable" subsets of $R \times S^{\prime}$ in the obvious way. It is easy to see that $\lambda^{*}$ is countably additive on $\mathcal{B}$, and that $\lambda^{*}$ still agrees with $\lambda_{1}$ on rectangles. In what follows, all subsets of $R \times S^{\prime}$ which are referred to will be understood to be in $B$.

Let $\mathcal{N}$ be the family of sets $H$ for which $\lambda^{*}(H)=0$, and let $E^{*}$ denote the $\sigma$-algebra $\mathcal{B}$ modulo $\mathcal{N}$. Clearly $\left(E^{*}, \lambda^{*}\right)$ is an $F^{\prime}$-measure algebra $\left({ }^{24}\right)$. To each

(24) The countable chain condition follows as in footnote 15 
$q \in P$ there corresponds the class of the "cylinder set" $q^{*} \times S^{\prime}$ in $E^{*}$, and we have $\lambda^{*}\left\{q^{*} \times S^{\prime}\right\}=\lambda_{1}\left\{q^{*} \times S^{\prime}\right\}=L(q)$. Thus (to within an isomorphism) $P$ is a subalgebra of $E^{*}$, and $\lambda^{*}$ agrees with $L$ on $P$; it follows that $E$ is a subalgebra of $E^{*}$, and $\lambda^{*}$ agrees with $\lambda$ on $E$. We have only to prove that $\left(E^{*}, \lambda^{*}\right)$ is full-valued.

First we note that, if $h \in E^{*}$ corresponds to $H \subset R \times S^{\prime}$, and if $x^{\prime} \in E^{\prime}$ corresponds to $x^{\prime *} \subset S^{\prime}$, then

$$
\lambda^{*}\left\{H \cap\left(R \times x^{*}\right)\right\}=\lambda^{*}(h) \chi\left(x^{\prime}\right),
$$

a result which follows at once from the definition of $\lambda^{*}$. We next prove:

(7) Given $H \subset R_{n} \times S^{\prime}$, and $f^{\prime} \in F^{\prime}$ such that $0<f^{\prime} \leqq \lambda^{*}(H)$, there exists $K \subset H$ such that $0<\lambda^{*}(K) \leqq f^{\prime}$.

For we have $f^{\prime} \geqq \epsilon \chi\left(x^{\prime}\right)$ for some $\epsilon>0$ and some nonzero $x^{\prime} \in E^{\prime}$. Since $L\left(R_{n}\right) \ll \infty$, there exists $y^{\prime} \neq o^{\prime}$ in $E^{\prime}$ such that $y^{\prime} \leqq x^{\prime}$ and $L\left(R_{n}\right) \ll$ some positive constant $c$ (say) on $y^{\prime *}$. Thus, from (6), $0<\lambda^{*}\left\{H \cap\left(R \times y^{\prime *}\right)\right\} \leqq c \chi\left(y^{\prime}\right)$. Choose $m>c / \epsilon$. From (1), we can find $m$ disjoint elements $q_{1}, q_{2}, \cdots, q_{m}$ in $P$ such that $\bigcup q_{m}^{*}=R_{n}$ and $L\left(q_{1}\right)=\cdots=L\left(q_{m}\right)=(1 / m) L\left(R_{n}\right)$. At least one of the rectangles $r_{i}=q_{i}^{*} \times y^{*}$ is such that $\lambda^{*}\left(H \cap r_{i}\right) \neq 0$, and we have only to take $K=H \cap r_{i}$.

Now if $h \in E^{*}$, corresponding to $H \subset R_{n} \times S^{\prime}$, is given, and if $0 \leqq f^{\prime} \leqq \lambda^{*}(h)$, there clearly exists a maximal $k \leqq h$ such that $\lambda^{*}(k) \leqq f^{\prime}$; and, from (7) (applied to $h-k$ and $f^{\prime}-\lambda^{*}(k)$ ), we must have $\lambda^{*}(k)=f^{\prime}$. Finally it is easy to drop the restriction $H \subset R_{n} \times S^{\prime}$ here, and this proves that $\left(E^{*}, \lambda^{*}\right)$ is fullvalued.

5.2. The theory of [5] now enables us to derive the structure of general $F^{\prime}$-measure algebras and $F^{\prime}$-integrals. For $F^{\prime}$-measures we have:

THEOREM 7. Every $F^{\prime}$-measure algebra is strictly $F^{\prime}$-isomorphic to a subalgebra of a principal ideal in a direct product $(J, m) \times E^{\prime}$ (with standard $F^{\prime}$-measure), where $(J, m)$ is a nonatomic ( $\sigma$-finite) measure algebra, and $F^{\prime}=F\left(E^{\prime}\right)$.

For, from Theorem 6 , the $F^{\prime}$-measure algebra $(E, \lambda)$ is strictly $F^{\prime}$-isomorphic to a subalgebra of a full-valued $F^{\prime}$-measure algebra $\left(E^{*}, \lambda^{*}\right)$, and, from Theorem $5(4.3),\left(E^{*}, \lambda^{*}\right)$ is strictly $F^{\prime}$-isomorphic to a principal ideal in a direct product of the kind described.

Since, conversely, every subalgebra of a principal ideal in $(J, m) \times E^{\prime}$ is an $F^{\prime}$-measure algebra, this theorem characterizes $F^{\prime}$-measure algebras.

5.3. For $F^{\prime}$-integrals we obtain the following theorem, which is the principal theorem of this paper.

THEOREM 8. Let $\phi$ be an $F^{\prime}$-integral on $F=F(E)$, where $F^{\prime}=F\left(E^{\prime}\right)$. Then $E$ is isomorphic to a subalgebra of a principal ideal in a direct product $(J, m)$ $\times E^{\prime}$, where $(J, m)$ is a nonatomic ( $\sigma$-finite) numerical measure algebra, and $\phi$ is strictly isomorphic (in the sense of 2.5) to the integral induced on $F$ (as in 


\section{4) by the standard $F^{\prime}$-integral $f \rightarrow \int f d M$ on this product (cf. 3.2).}

That is, given an $F^{\prime}$-integral $\phi$ on $F=F(S, \mathcal{B}, \mathcal{N})$ with values in $F\left(S^{\prime}, \mathcal{B}^{\prime}, \mathcal{N}^{\prime}\right)$, there exist (i) a (numerical) measure space $\left(X, \mathcal{B}_{m}, \mathcal{N}_{m}\right)$ with a ( $\sigma$-finite, nonatomic) measure $m$, (ii) a "measurable" subset $S_{0}$ of the product space $X \times S^{\prime}$ (with suitable definitions of "measurable" and "null" sets in this product), (iii) a sub-field $\mathcal{B}_{0}$ of the "measurable" subsets of $S_{0}$, (iv) an isomorphism $\theta$ of $B \bmod \mathcal{N}$ onto $B_{0} \bmod \mathcal{N}_{0}$, where $\mathcal{N}_{0}$ consists of the "null" sets in $\mathcal{B}_{0}$. And $\theta$ induces (cf. 1.6) a strict isomorphism $\psi$ of $F$ onto the subspace $F\left(S_{0}, \mathcal{B}_{0}, \mathcal{N}_{0}\right)$ of those functions on $X \times S^{\prime}$ which vanish outside $S_{0}$ and are "measurable $\mathcal{B}_{0}$," in such a way that, for each integrable $f \in F$ (in particular, for each $f \geqq 0), \phi(f)$ is $\left(\bmod \mathcal{N}^{\prime}\right)$ the function on $S^{\prime}$ whose value at $s^{\prime}$ is (from 3.2) $\int_{X}(\psi f)\left(x, s^{\prime}\right) d m(x)$. In a sense, this gives a complete description of the general $F^{\prime}$-integral.

To prove the theorem, we have only to write $\lambda(x)=\phi(\chi(x))(x \in E)$, and apply Theorem 7 to $(E, \lambda)$. After an isomorphism of $E$ we obtain $E$ as a subalgebra of a principal ideal in $(J, m) \times E^{\prime}$, and have $\lambda(x)=M(x)(x \in E)$. It follows from 2.3 that $\phi(f)=\int f d M$ for all "integrable" $f \in F$.

REMARK. If the $F^{\prime}$-measure $\lambda$ induced by $\phi$ is full-valued, we can use Theorem 5 (4.5) directly, instead of Theorem 7, in deriving Theorem 8. This means that the words "sub-algebra of" can now be omitted in Theorem 8i.e., in the realization described above, $\mathcal{B}_{0}$ may now be taken to consist of all "measurable" subsets of $S_{0}$.

5.4. Some examples. To illustrate Theorem 8 , we consider two simple examples. First let $F$ be the space of measurable functions (modulo null sets) $f(x, y)$ of two real variables, with the usual plane measure, and let $F^{\prime}$ similarly be the space of measurable functions on the line. Let $k(x, y)$ be measurable, positive, and finite for almost all $(x, y)$, and define $\phi(f)=f^{\prime}$ where $f^{\prime}(x)$ $=\int_{-\infty}^{\infty} k(x, y) f(x, y) d y$, whenever this integral exists unambiguously. (It may be infinite.) Then $\phi$ is an $F^{\prime}$-integral on $F$; this is most easily verified by noting that $\phi$, restricted to non-negative elements of $F$, satisfies the requirements $(\alpha),(\beta),(7)$, and (8) of 2.1. In the present case it is not hard to see that $\lambda$ is full-valued, so the construction leading to Theorem 8 can here be simplified. If we assume further that $\int_{-n}^{n} k(x, y) d y$ is finite for all $n(=1,2, \cdots)$ and almost all $x$ (this involves no essential loss of generality, as it can always be achieved by a preliminary measure-preserving transformation of the plane onto itself, leaving invariant every line $x=$ constant), a suitable representation can be described very simply. Define $z=z(x, y)$ by: $\int_{0}^{z} k(x, t) d t$ $=y$; then $\phi(f)=f^{\prime}$ where $f^{\prime}(x)=\int_{-\infty}^{\infty} f(x, z) d y$ (for almost all $x$ ), so that the mapping $(x, y) \rightarrow(x, z)$ gives the desired isomorphic imbedding of the plane in $(J, m) \times E^{\prime}$-i.e., in itself.

For a second example, take $F=F^{\prime}=$ space of measurable functions (modulo null sets) on the line, and, with the same assumptions on $k(x, y)$ as before, let $\phi(f)=f^{\prime}$ where $f^{\prime}(x)=\int_{-\infty}^{\infty} k(x, y) f(y) d y$. Again $\phi$ is an $F^{\prime}$-integral 
on $F$, but $\lambda$ is in general no longer full-valued. However, in the present case it suffices to multiply $(E, \lambda)$ by $E^{\prime}$ to obtain $(E, \lambda)$ imbedded in a full-valued $F^{\prime}$-measure algebra. This comes to regarding $F$ as imbedded in the space of functions of two variables, $f$ corresponding to $\bar{f}$ where $\bar{f}(x, y)=f(y)$ (independent of $x$ ), and we obtain $\phi(\bar{f})=f^{\prime}$ where $f^{\prime}(x)=\int_{-\infty}^{\infty} k(x, y) \bar{f}(x, y) d y$. Thus this second example is imbedded in the first.

\section{Full-valued $F^{\prime}$-INTEGRALS}

6.1. We have seen that, if the $F^{\prime}$-measure $\lambda$ induced by an $F^{\prime}$-integral $\phi$ on $F=F(E)$ happens to be full-valued, the description of $\phi$ provided by Theorem 8 can be made more precise; $E$ can be realized as a principal ideal of $(J, m) \times E^{\prime}$, and not merely as a subalgebra of such a principal ideal. In this section we investigate a simple condition on $\phi$ which (roughly speaking) ensures that $\lambda$ is "nearly" full-valued and so enables a more precise description to be given.

We define an $F^{\prime}$-integral $\phi$ on $F$ to be "full-valued" if it has the property:

(1) Given $f \in F$ such that $f \geqq 0$, and $g^{\prime} \in F^{\prime}$ such that $0 \leqq g^{\prime} \leqq \phi(f)$, there exists $g \in F$ such that $0 \leqq g \leqq f$ and $\phi(g)=g^{\prime}$.

Clearly if $\phi$ is numerical-i.e., if $E^{\prime}$ is a single atom-then $\phi$ is full-valued. It is also easy to see that if the induced $F^{\prime}$-measure $\lambda$ is full-valued (4.1(1)) then so is $\phi$. The converse is false, since if $\phi$ is a strict isomorphism of $F$ it is automatically full-valued, but the values of $\lambda$ are then restricted to characteristic functions. The following theorem shows, however, that this is essentially the only case in which this converse fails.

THEOREM 9. Let $\phi$ be an $F^{\prime}$-integral on $F$, where $F=F(E)$ and $F^{\prime}=F^{\prime}\left(E^{\prime}\right)$. Then the following statements are equivalent.

$(\alpha) \phi$ is full-valued.

( $\beta) E^{\prime}$ is isomorphic to a subalgebra $A$ of $E$ in such a way that, if $g \in F(A)$ (considered as a subspace of $F$ ) corresponds to $g^{\prime} \in F^{\prime}$ under this isomorphism, we have $\phi(f g)=\phi(f) g^{\prime}$ whenever $f \in F$ and $f, f g$ are both integrable.

$(\gamma) E$ can be expressed as the direct sum of (at most) countably many principal ideals $E_{*}, D_{1}, D_{2}, \cdots\left({ }^{25}\right)$, in such a way that: (i) for each $n, \phi$ is an isomorphism of $F\left(D_{n}\right)$ onto a subspace of $F^{\prime}$ corresponding to a principal ideal in $E^{\prime}$, and (ii) on $E_{*}$, the $F^{\prime}$-measure $\lambda$ induced by $\phi$ is full-valued.

REMARKs. To avoid circumlocution, $F(A)$ and $F\left(D_{n}\right)$ are here identified with the subspaces of $F(E)$ which are naturally isomorphic to them (i.e., they are imbedded in $F$ as in 2.4), and we speak of $\lambda$ on $E_{*}$ as an $F^{\prime}$-measure even though it may happen that $\lambda\left(e_{*}\right)$ fails to be $\gg 0$; strictly speaking, $\lambda$ on $E_{*}$ will be an $F^{\prime \prime}$-measure, where $F^{\prime \prime}$ is the subspace of $F^{\prime}$ determined by the principal ideal $E_{*}^{\prime}$ of $\left[\lambda\left(e_{*}\right)\right]$ in $E^{\prime}$.

${ }^{25}$ That is, the unit element $e$ of $E$ can be written as $e_{*} \bigvee \bigvee d_{n}$ where the elements $e_{*}, d_{n}$ are disjoint, $E_{*}$ is the principal ideal $E\left(e_{*}\right)$, and similarly $D_{n}=E\left(d_{n}\right)$. 
Note that, in $(\beta)$, we have $\phi(g)=\phi(1) g^{\prime}(g \in F(A))$; but $\phi$ need not itself be an isomorphism between $F(A)$ and $F\left(E^{\prime}\right)$, as $\phi(1)$ may be infinite. It is easy to see that, in $(\beta), f g$ is integrable whenever $f$ is integrable and $g \in F(A)$ is finite.

Finally we remark that, in $(\gamma)$, Theorem $5(4.3)$ can be applied to $E_{*}$, giving:

CoRollary. $E_{*}$ is isomorphic to a principal ideal in a direct product $(J, m) \times E_{*}^{\prime}$, where $(J, m)$ is a nonatomic measure algebra and $E_{*}^{\prime}$ is a principal ideal in $E^{\prime}$; and $\phi$ on $F\left(E_{*}\right)$ is then induced by the standard integral $\int f d M$ on $(J, m) \times E_{*}^{\prime}$.

The effect of $\phi$ on all $F$ is thus determined in $(\gamma)$, since every $f \in F$ is uniquely expressible as $f_{*}+\sum g_{n}$ where $f_{*} \in F\left(E_{*}\right)$ and $g_{n} \in F\left(D_{n}\right)$.

6.2. Proof that $(\alpha)$ implies $(\beta)$. In this paragraph we make the convention that $\infty-\infty=0$. With this understanding, it is easy to verify that, for any $F^{\prime}$-measure $\lambda$, we have

$$
\lambda(x)-\lambda(y) \leqq \lambda(x-y), \quad \text { provided } y \leqq x(\in E) .
$$

For each $x \in E$ write $[\lambda(x)]=\theta(x)\left(\in E^{\prime}\right)$; to simplify the notation, we shall write $\theta([f])$, where $f \in F$, as $\theta[f]$. Then

$$
\theta[f]=[\phi(f)] \text { if } f \geqq 0,
$$

for if $[f]=y$ we have $\infty \cdot f=\infty \cdot \chi(y)$, and so $\infty \phi(f)=\infty \lambda(y)$, giving (3). Now, given $x \in E$ and $x^{\prime} \in E^{\prime}$, we define

$$
\pi\left(x, x^{\prime}\right)=\bigvee\left\{y \mid y \in E, y \leqq x, \text { and } \theta(y) \leqq x^{\prime}\right\} .
$$

We shall prove

Lemma 4. If $\phi$ is full-valued, the correspondence $x^{\prime} \rightarrow \pi\left(e, x^{\prime}\right)$ is a $\sigma$-isomorphism of $E^{\prime}$ onto a subalgebra $A$ of $E$; the inverse isomorphism is $\theta$. Further, for all $x \in E$ and $x^{\prime} \in E^{\prime}, \lambda\left(x \pi\left(e, x^{\prime}\right)\right)=\lambda(x) \chi\left(x^{\prime}\right)$.

The first step is to show

$$
\lambda\left(\pi\left(x, x^{\prime}\right)\right)=\lambda(x) \chi\left(x^{\prime}\right) \text { (provided } \phi \text { is full-valued). }
$$

Write $\pi\left(x, x^{\prime}\right)=\pi$; the countable chain condition gives $\pi=V_{y_{n}}$ $(n=1,2, \cdots)$ where $y_{n} \leqq x$ and $\theta\left(y_{n}\right) \leqq x^{\prime}$, whence $\lambda\left(y_{n}\right) \leqq \infty \chi\left(x^{\prime}\right)$. Thus $\pi \leqq x$ and $\lambda(\pi) \leqq \infty \chi\left(x^{\prime}\right)$, and so $\lambda(\pi) \leqq \inf \left\{\lambda(x), \infty \chi\left(x^{\prime}\right)\right\}=\lambda(x) \chi\left(x^{\prime}\right)$. Write $h^{\prime}=\lambda(x) \chi\left(x^{\prime}\right)-\lambda(\pi)$ (with the convention introduced above); then $h^{\prime} \geqq 0$, and we must prove $h^{\prime}=0$. Now $h^{\prime} \leqq \lambda(x-\pi)$, by (2); hence from (1) there exists $h \leqq \chi(x-\pi)$ such that $h \geqq 0$ and $\phi(h)=h^{\prime}$. Let $[h]=y$; then $y \leqq x$, and (from (3)) $\theta(y)=\left[h^{\prime}\right] \leqq x^{\prime}$, and so $y \leqq \pi$. But $y \leqq x-\pi$; thus $y=o$, and therefore $h$ and $h^{\prime}$ vanish.

It follows from (5) that 


$$
\theta\left(\pi\left(x, x^{\prime}\right)\right)=x^{\prime} \theta(x) .
$$

In particular, $\theta\left(\pi\left(e, x^{\prime}\right)\right)=x^{\prime}$; hence the mapping $x^{\prime} \rightarrow \pi\left(e, x^{\prime}\right)$ of $E^{\prime}$ in $E$ is $1-1$, with inverse $\theta$, and clearly this mapping maps $o^{\prime}$ on $o$ and $e^{\prime}$ on $e$. To show that it is an isomorphism of $E^{\prime}$ onto a subalgebra (say) $A$ of $E$, we have only to show that it preserves (countable) suprema and disjointness. Suppose, then, that $\pi\left(e, x_{n}^{\prime}\right)=\pi_{n}, \bigvee x_{n}^{\prime}=x^{\prime}$, and $\pi\left(e, x^{\prime}\right)=\pi ;$ clearly $\pi \geqq \pi_{n}$ and so $\pi \geqq \bigvee \pi_{n}$. Let $t=\pi-\mathrm{V}_{n}$, and suppose $t \neq 0$. Using (5), we have $\lambda(t) \leqq \lambda(\pi)=\lambda(e) \chi\left(x^{\prime}\right)$ $\leqq \sum \lambda(e) \chi\left(x_{n}^{\prime}\right)$; and since $\lambda(t) \neq 0$ there exists $n$ such that inf $\left\{\lambda(t), \lambda(e) \chi\left(x_{n}^{\prime}\right)\right\}$ $=g^{\prime}$ (say) $\neq 0$. From (1), there exists $g \in F$ such that $0 \leqq g \leqq \chi(t)$ and $\phi(g)=g^{\prime}$. Let $y=[g]$; then, from $(3), \theta(y)=\left[g^{\prime}\right] \leqq x_{n}^{\prime}$, and therefore $y \leqq \pi_{n}$; but $y \leqq t$, whence $y=o$ and $g$ and $g^{\prime}$ must vanish, giving a contradiction. Thus $\pi\left(e, \bigvee x_{n}^{\prime}\right)=\bigvee \pi\left(e, x_{n}^{\prime}\right)$. Again, if $x^{\prime} y^{\prime}=o^{\prime}$, let $t=\pi\left(e, x^{\prime}\right) \pi\left(e, y^{\prime}\right)$; from (6), $\theta(t) \leqq x^{\prime} y^{\prime}$, so $t=0$.

Again, for any $x \in E$ and $x^{\prime} \in E^{\prime}$, we have $\pi\left(x, x^{\prime}\right) \leqq \pi\left(e, x^{\prime}\right)$ and $\pi\left(x, x^{\prime}\right)$ $\leqq x$, so that $\pi\left(x, x^{\prime}\right) \leqq x \pi\left(e, x^{\prime}\right)$. On the other hand, (6) gives $\theta\left(x \pi\left(e, x^{\prime}\right)\right)$ $\leqq x^{\prime}$, and so (from (4)) $x \pi\left(e, x^{\prime}\right) \leqq \pi\left(x, x^{\prime}\right)$. Hence $\pi\left(x, x^{\prime}\right)=x \pi\left(e, x^{\prime}\right)$, and so $\lambda\left(x \pi\left(e, x^{\prime}\right)\right)=\lambda(x) \chi\left(x^{\prime}\right)$, from $(5)$.

On applying the isomorphism given by Lemma 4 , we may suppose that $E^{\prime}$ is identical with $A$, and that $\lambda\left(x x^{\prime}\right)=\lambda(x) \chi\left(x^{\prime}\right)$ for all $x \in E$ and $x^{\prime} \in A$. It follows from Lemma 1 (1.4) that if $f \in F, g \in F(A)$, and $f, g$ are both nonnegative, then $\phi(f g)=\phi(f) g$; and $(\beta)$ follows easily.

6.3. Now suppose $\phi$ has property $(\beta)$ of Theorem 9 ; we prove it has $(\gamma)$. We may again take $E^{\prime}=A$, and so have

$$
\lambda\left(x x^{\prime}\right)=\lambda(x) \chi\left(x^{\prime}\right) \quad\left(x \in E, x^{\prime} \in A \subset E\right) .
$$

For each $x \in E$ we write $\bar{x}$ (or $\mathrm{Cl}(x)$ ) for the smallest element of $A$ which contains $x$. (It is easy to see that $\bar{x}=\theta(x)$, in the notation of 6.2.) The obvious property

(8) If $a \in A$ and $a x=0$, then $a \bar{x}=0$, will often be useful.

From $[6, \S 3]\left({ }^{26}\right)$, the unit-element $e$ of $E$ can be expressed as a supremum of disjoint elements, $e=e_{*} \bigvee \bigvee d_{n}$, where $\bigvee d_{n}$ is the supremum of all elements of $E$ which are of order 0 over $A\left({ }^{27}\right)$, each $d_{n}$ is of order 0 over $A$, and $\bar{d}_{1} \geqq \bar{d}_{2} \geqq \cdots$ As in [6], the ideals $E\left(d_{n}\right)$ and $A\left(\bar{d}_{n}\right)$ are isomorphic under the correspondence $x\left(\leqq d_{n}\right) \rightarrow \bar{x}$. If $x \leqq d_{n}$, then $d_{n} \bar{x}=x$ since $d_{n}$ is of order 0 (cf. $[6,3.2(3)])$, and so (7) gives

$$
\lambda(x)=\lambda\left(d_{n}\right) \chi(\bar{x}) \text { if } x \leqq d_{n} .
$$

${ }^{(26)}$ In [6] it was assumed that $E$ was a measure algebra, but this assumption plays no part in the results quoted here.

(27) An element $x \in E$ is of order 0 over $A$ if the ideal $E(x)$ coincides with the set $x A$ of elements of the form $x a, a \in A$. For the general definition see [5, p. 301]. 
It follows that if $[f] \leqq d_{n}$-i.e., in effect, if $f \in F\left(D_{n}\right)$ where $D_{n}=E\left(d_{n}\right)$-we have

$$
\phi(f)=\lambda\left(d_{n}\right) f^{\prime},
$$

where $f^{\prime}$ is the function in $F\left(A\left(\bar{d}_{n}\right)\right)$ which corresponds to $f$ under the natural isomorphism $x \rightarrow \bar{x}$ between $D_{n}$ and $A\left(\bar{d}_{n}\right)$. Thus to show that $\phi$ maps $F\left(D_{n}\right)$ isomorphically onto the subspace $F\left(A\left(\bar{d}_{n}\right)\right)$ of $F^{\prime}$, we have only to show that $0 \ll \lambda\left(d_{n}\right) \ll \infty$ on $\bar{d}_{n}$. Now $2.1(6)$ gives $d_{n}=\mathrm{V}_{m} c_{n m}$, say, where $\lambda\left(c_{n m}\right) \ll \infty$, and the elements $c_{n m}$ can be taken to be disjoint. But since $d_{n}$ is of order 0 over $A$, it follows from [6, 3.2(3)] that the elements $\mathrm{Cl}\left(c_{n m}\right)$, for fixed $n$, are also disjoint. Thus, from (9), the finite functions $\lambda\left(c_{n m}\right)$ have (for fixed $n$ ) disjoint loci, and therefore their sum $\lambda\left(d_{n}\right)$ is everywhere finite. On the other hand, if $x^{\prime}=-\left[\lambda\left(d_{n}\right)\right]$, (7) gives $d_{n} x^{\prime}=o$, and therefore $\bar{d}_{n} \leqq\left[\lambda\left(d_{n}\right)\right]$, which shows that $\lambda\left(d_{n}\right) \gg 0$ on $\bar{d}_{n}$. This has established part (i) of $(\gamma)$.

6.4. For the rest of the proof that $(\beta)$ implies $(\gamma)$, we shall be concerned only with the ideal $E_{*}$ and with $F\left(E_{*}\right)$, on which the values of $\phi$ are always in $F\left(A\left(\bar{e}_{*}\right)\right)$. Now the ideal $A\left(\bar{e}_{*}\right)$ is isomorphic (under the correspondence $\left.a \rightarrow e_{*} a\right)$ to the subalgebra $e_{*} A\left(\tilde{e}_{*}\right)$ of $E_{*}$. Hence we may simplify the notation by omitting the asterisks, regarding $e_{*}$ as $e$ and $e_{*} A\left(\bar{e}_{*}\right)$ as $A$, and have (after another isomorphism of $E^{\prime}$ ) that (7) above still holds and that $E$ now contains no nonzero element of order 0 over the subalgebra $A\left(=E^{\prime}\right)$ of $E$. We must deduce that $(E, \lambda)$ is full-valued. First we show:

(11) If $x \in E$, and $x \neq o$, there exists $y \leqq x$ such that

$$
0<\lambda(y) \leqq(1 / 2) \lambda(x) .
$$

We may assume $\lambda(x) \ll \infty$, since otherwise we can replace $x$ (using 3.1(4)) by a smaller nonzero element of finite $\lambda$. Since $x$ is not of order 0 over $A$, there exists $z<x$ not of the form $x a, a \in A$. Hence $0<\lambda(z)<\lambda(x \bar{z})=\lambda(x) \chi(\bar{z})$, by (7). Let $b=[\lambda(x) \chi(\bar{z})-\lambda(z)]$; then $b \in A, b \leqq \bar{z}$, and $b \neq o$. Further, since $b \leqq[\lambda(x-z)] \leqq \mathrm{Cl}(x-z)$, from $(7)$, we have $b(x-z) \neq 0$.

Using any realization of $A$, let $c \in A$ correspond to the "subset" of $b$ where $\lambda(z) \leqq(1 / 2) \lambda(x)$. Define $y=c z \bigvee(b-c)(x-z)$. We have $\lambda(c z)=\lambda(z) \chi(c)$ $\leqq(1 / 2) \lambda(x) \chi(c)$, and $\lambda\{(b-c)(x-z)\}=\lambda(x-z) \chi(b-c) \leqq(1 / 2) \lambda(x) \chi(b-c)$; hence $\lambda(y) \leqq(1 / 2) \lambda(x) \chi(b) \leqq(1 / 2) \lambda(x)$. Finally, $y \neq o$, since if $c \neq 0$ then (as $c \leqq \bar{z}) c z \neq 0$, while if $c=0$ then $(b-c)(x-z)=b(x-z) \neq 0$.

Iteration of (11) gives:

(12) If $x \neq o$, there exists $y \leqq x$ such that $0<\lambda(y) \leqq\left(1 / 2^{n}\right) \lambda(x)$.

We next deduce:

(13) If $x \in E, f^{\prime} \in F^{\prime}$, and $0<f^{\prime} \leqq \lambda(x)$, there exists $y \leqq x$ such that $0<\lambda(y)$ $\leqq f^{\prime}$.

Again it suffices to prove this assuming $\lambda(x) \ll \infty$. Since $f^{\prime}>0$, there exist a nonzero element $a \in E$, and a positive constant $\epsilon$, such that $f^{\prime} \geqq \epsilon \chi(a)$. There exist a nonzero element $b \in A$, and a positive constant $k$, such that $b \leqq a$ 
and $\lambda(x) \chi(b) \leqq k \chi(b)$; thus, from (7), $\lambda(x b) \leqq k \chi(b)$. From (12) we obtain an element $y \leqq x b$ such that $0<\lambda(y)<\epsilon \chi(b) \leqq f^{\prime}$.

By a standard argument, choosing $y$ to be maximal in (13) (cf. end of 5.1), we obtain $\lambda(y)=f^{\prime}$ in (13), which proves that $\lambda$ is full-valued.

6.5. Finally, $(\gamma)$ implies that $\phi$ is full-valued. For $\phi$ is necessarily fullvalued on each $F\left(D_{n}\right)$ and on $F\left(E_{*}\right)$, and it readily follows that $\phi$ is fullvalued on $F(E)$. This completes the proof of Theorem 9 .

We remark further that, for any $F^{\prime}$-integral $\phi$ on any $F(E)$, where $F^{\prime}$ $=F\left(E^{\prime}\right)$, the corresponding $F^{\prime}$-integral on the space of functions on the "direct product" $(E, \lambda) \times E^{\prime}$ (cf. 5.1) is always full-valued. This can be proved by "decomposing" this direct product with respect to its subalgebra $E^{\prime}$ (cf. $[6,3.2(5)])$ and showing that this leads to a decomposition of $\phi$ of the type in Theorem $9(\gamma)$. Hence, for the purpose of getting a representation for the general $F^{\prime}$-integral, the construction in 5.1 can be simplified by omitting the multiplication by $(I, \mu)$. This imbeds $E$ in an algebra on which the corresponding $F^{\prime}$-integral (but not in general the $F^{\prime}$-measure) is full-valued; and Theorem 9 then leads to a description of $\phi$ which is more economical, though more complicated, than that of Theorem 8 (5.3).

\section{Homogeneous $F^{\prime}$-INTEGRALS}

7.1. The condition of full-valuedness considered in $\$ 6$ is quite strong. We shall now show that a somewhat weaker, and quite natural, condition would suffice, provided that we are prepared to change the function-space $F^{\prime}$ of values of the integral.

Definition. An $F^{\prime}$-integral $\phi$ on $F$ will be called "homogeneous" if it satisfies:

(1) If $f, g$ are non-negative elements of $F$ such that $\phi(f)=\phi(g)$, then, given $f_{1} \in F$ such that $0 \leqq f_{1} \leqq f$, there exists $g_{1} \in F$ such that $0 \leqq g_{1} \leqq g$ and $\phi\left(g_{1}\right)=\phi\left(f_{1}\right)$.

This condition is very similar to that imposed on "abstract measures" in [5]; see 3.3(II). There is in fact a connection between the two conditions, but not a direct one. If $\phi$ satisfies (1), the corresponding $F^{\prime}$-measure $\lambda$ (where $\lambda(x)=\phi(\chi(x))$ need not satisfy $3.3($ II), and if $\lambda$ satisfies 3.3 (II), $\phi$ need not satisfy (1). This is shown by the following simple examples. In both examples we take $E$ to be realized by the unit square $0 \leqq x, y \leqq 1$ in the plane, together with the segment $0 \leqq x \leqq 1, y=2$, with plane measure in the square and linear measure on the segment. In both cases, $E^{\prime}$ will be realized by the unit segment $0 \leqq x \leqq 1$, with linear measure. For the first example, define $\phi(f)=f^{\prime}$ where $f^{\prime}(x)=\int_{0}^{1} f(x, y) d y+f(x, 2)$. It can be verified that $\phi$ is a homogeneous $F^{\prime}$. integral on $F=F(E)$, where $F^{\prime}=F\left(E^{\prime}\right)$; but $\lambda$ does not satisfy 3.3(II). For the second example, define $\phi(f)=f^{\prime}$ where $f^{\prime}(x)=\int_{0}^{1} f(x, y) d y+(1 / x) \int_{0}^{1} f(t, 2) d t$. The corresponding $\lambda$ is an $F^{\prime}$-measure on $E$ and satisfies 3.3(II) (in fact, $(E, \lambda)$ is an "abstract measure algebra"), but it is easy to see that $\phi$ is not homogeneous. 
However, it can be shown that if $\phi$ is homogeneous, $E$ is expressible as a direct sum of a sequence of principal ideals on each of which $\lambda$ satisfies 3.3(II). This will follow from Theorem 10 below; we can replace $\phi$ by a full-valued integral without changing the " $\sim$ " relation (3.3), and Theorem 9 (6.1) then gives the desired decomposition. Similarly, if $\lambda$ satisfies 3.3(II), $E$ is expressible as a direct sum of principal ideals $E_{n}$ such that $\phi$ is homogeneous on each $F\left(E_{n}\right)$ (with values in a suitable subspace of $F^{\prime}$ ); this can be shown by applying [5] to decompose $E$ into principal ideals on each of which $\lambda$ is either "trivial" or full-valued.

7.2. We shall prove:

THEOREM 10. If $\phi$ is a homogeneous $F^{\prime}$-integral on $F=F(E)$, there exists a full-valued $F^{\prime \prime}$-integral $\phi^{\prime}$ on $F$ which refines $\phi$ (in the sense of 2.5); and $\phi^{\prime}$ is unique, to within isomorphism. Conversely, every $F^{\prime}$-integral which has a fullvalued refinement must be homogeneous.

Thus the statements $(\beta)$ and $(\gamma)$ of Theorem 9 (6.1) will apply to $\phi^{\prime}$, and hence to the " " relation induced by $\phi(3.3)$, since this is unchanged

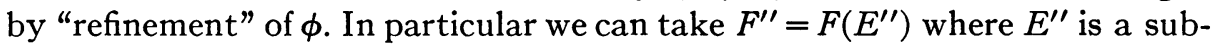
algebra of $E$; I do not know whether $E^{\prime \prime}$ will always be isomorphic to a subalgebra of $E^{\prime}$, where $F^{\prime}=F\left(E^{\prime}\right)$.

The proof of Theorem 10 is quite long, and we shall omit many details. The procedure will be to construct first a suitable algebra $A\left(=E^{\prime \prime}\right)$ of $E$, and to show that, given $g \in F$, with $g \geqq 0$, there exists a unique $h \in F$ which is "measurable $A$ " and such that $h \geqq 0$ and $\phi(g)=\phi\left(f_{0} h\right), f_{0}$ being a suitable (fixed) element of $F$. We then define (roughly speaking) $\phi^{\prime}(g)=h$, and show that $\phi^{\prime}$ has the desired properties. Its uniqueness will follow from Theorem 9. The converse part of Theorem 10 is trivial, so we assume $\phi$ homogeneous throughout what follows.

7.3. For each $x \in E$, write $\tau(x)=\pi(e, \theta(x))$ in the notation of 6.2 ; that is, $\tau(x)=\mathrm{V}\{y \mid[\lambda(y)] \leqq[\lambda(x)]\}$, where $\lambda(x)=\phi(\chi(x))$. Clearly $\tau(x) \geqq x$, and if $x<y$ then $\tau(x) \leqq \tau(y)$. Also, from the countable chain condition, $\theta(\tau(x))=\theta(x)$, and so $\tau(\tau(x))=\tau(x)$.

Lemma 5. Let $A=\{\tau(x) \mid x \in E\}$. Then $A$ is a ( $\sigma-)$ subalgebra of $E$, and $\tau(x)$ is the smallest element of $A$ containing $x{ }^{(28)}$.

Suppose $a_{n} \in A(n=1,2, \cdots)$ and $\bigvee a_{n}=b$; we prove $\tau(b)=b$ (and so $b \in A$ ). It will suffice to show that, whenever $x \leqq \tau(b)$ and $\lambda(x) \ll \infty$, then $x \leqq b$. Since $\theta(x) \leqq \theta(\tau(b))=\theta(b)$, we have $\phi(\chi(\mathbf{x})+\infty \chi(b))=\phi(\infty \chi(b))$; hence, by (1), there exists $h \in F$ such that $0 \leqq h \leqq \infty \chi(b)$ and $\phi(h)=\lambda(x)$. Writing $h_{n}$ $=h \chi\left(a_{n}-\bigvee_{i=1}^{n-1} a_{i}\right)$, we have $h=\sum h_{n}$. From (1) applied to $\phi(h)=\phi(\chi(x))$,

${ }^{(28)}$ If $\phi$ is full-valued, $A$ coincides with the algebra constructed in 6.2. 
there exists $k_{1} \in F$ such that $0 \leqq k_{1} \leqq \chi(x)$ and $\phi\left(k_{1}\right)=\phi\left(h_{1}\right)$. Similarly there exists $k_{2} \in F$ such that $0 \leqq k_{2} \leqq \chi(x)-k_{1}$ and $\phi\left(k_{2}\right)=\phi\left(h_{2}\right)$; and so on. Thus $\sum k_{n} \leqq \chi(x)$, and $\phi\left(\sum k_{n}\right)=\sum \phi\left(h_{n}\right)=\phi(h)=\phi(\chi(x)) \ll \infty$, so $\sum k_{n}=\chi(x)$, and therefore $x=\mathrm{V}\left[k_{n}\right]$. But $\left[h_{n}\right] \leqq a_{n}$, so $\theta\left(a_{n}\right) \geqq \theta\left[h_{n}\right]=\theta\left[k_{n}\right]$ (this last equality holding from $6.2(3))$, and hence $\left[k_{n}\right] \leqq \tau\left(a_{n}\right)=a_{n}$. Thus $x \leqq \bigvee a_{n}=b$.

By similar arguments one proves $\tau(-a)=-\tau(a)$, and that if $\tau(y) \geqq x$ then $\tau(y) \geqq \tau(x)$; and the lemma follows.

Convention. Throughout the rest of the proof, till 7.7, we make the convention that all elements $f, g$, etc., of $F$ referred to are to be non-negative. With this understanding, the following results follow easily from Lemma 5 and methods similar to those used in its proof.

(2) $\tau(x \bigvee y)=\tau(x) \bigvee \tau(y)$

(3) If $a \in A, \tau(x a)=a \tau(x)$.

(4) If $\tau(x) \leqq \tau(y)$, and $[f] \leqq x$, there exists $g$ such that $[g] \leqq y$ and $\phi(f)$ $=\phi(g)$.

(5) If $[\phi(f)]=[\phi(g)], \tau[f]=\tau[g]\left({ }^{29}\right)$.

(6) If $\phi(f)=\phi(g)$, and $a \in A$, then $\phi(f \chi(a))=\phi(g \chi(a))$.

7.4. Boundedness. The next group of results is concerned with a notion of "boundedness" similar to, but distinct from, the property of being bounded qua function on $S$ in a realization of $F$ as $F(S, \mathbb{B}, \mathcal{N})$; no confusion should arise, as we shall never have to consider boundedness in the latter sense. We define $f \in F$ to be bounded if the relations $f=g+h$ and $\phi(f)=\phi(g)$ (and, of course, $f, g, h \geqq 0$, in accordance with the above convention) together imply $h=0$. It is easy to see that $f$ is bounded if and only if (i) $f \ll \infty$, and (ii) $f \geqq g$ and $\phi(f)=\phi(g)$ imply $f=g$. If $\phi(f) \ll \infty, f$ is bounded, but the converse is false, in general (as can be seen from the example in 7.9 below). Hence (as is also clear directly) if $\phi(f) \ll \infty$, then also $f \ll \infty$. The proofs of the following results are easy.

(7) If $\phi(f)=\phi(g)$, and $f$ is bounded, then so is $g$.

(8) If $f \leqq g$, and $g$ is bounded, then so is $f$.

(9) If $f$ is bounded, and $\rho$ is a positive (finite) real number, then $\rho f$ is bounded.

(10) If $f_{n}$ is bounded $(n=1,2, \cdots)$, and the elements $\tau\left[f_{n}\right]$ are (pairwise) disjoint, then $\sum f_{n}$ is bounded.

For let $\sum f_{n}=f=g+h$, where $\phi(f)=\phi(g)$; we must prove $h=0$. Write $\tau\left[f_{n}\right]=a_{n}(\in A), g_{n}=g \chi\left(a_{n}\right)$, and $h_{n}=h \chi\left(a_{n}\right)$; then $g_{n}+h_{n}=f \chi\left(a_{n}\right)=f_{n}$, and (from (6) above) $\phi\left(g_{n}\right)=\phi\left(f_{n}\right)$, and so $h_{n}=0$ for all $n$ because $f_{n}$ is bounded. But $h=\sum h_{n}=0$.

(11) There exists a bounded $f_{0}$ such that $\tau\left[f_{0}\right]=e$.

Let $\left\{g_{n}\right\}$ be a maximal collection (necessarily countable) of bounded elements of $F$ such that the elements $\tau\left[g_{n}\right]$ of $E$ are disjoint and nonzero. Let $f_{0}=V g_{n}$; by (10), $f_{0}$ is bounded. If $\tau\left[f_{0}\right] \neq e$, there exists a nonzero $a \in A$

${ }^{(29)}$ We write $\tau[f]$ as an abbreviation for $\tau([f])(f \in F)$. 
disjoint from every $\tau\left[g_{n}\right]$. Now (2.1(6)) $e=\mathrm{V}_{x_{n}}$ where $\phi\left(\chi\left(x_{n}\right)\right) \ll \infty$, and for some $n$ we have $x_{n} a \neq 0$. Hence, defining $g_{0}=\chi\left(x_{n} a\right)$, we have that $g_{0}$ is bounded and $o \neq\left[g_{0}\right] \leqq a$, and so $o \neq \tau\left[g_{0}\right] \leqq a$, contradicting the maximality of $\left\{g_{n}\right\}$.

REMARK. It follows that $\phi\left(f_{0}\right) \gg 0$.

(12) Given $f, h$, such that $\tau[h] \leqq \tau[f]$ and $f$ is bounded; if, for every positive real number $\rho$, there exists $k_{\rho} \leqq \rho f$ such that $\phi(h)=\phi\left(k_{\rho}\right)$, then $h=0$.

Taking $\rho=1 / n$ and $h_{n}=n k_{\rho}$, we obtain $h_{n} \leqq f$ such that $\phi\left(h_{n}\right)=n \phi(h)$ $(n=1,2, \cdots)$. Let $h^{*}=\sup h_{n}$; then $\phi\left(h^{*}\right) \geqq \infty \phi(h)$, and so $\phi\left(h^{*}\right)=\phi\left(h^{*}+h\right)$. If $h \neq 0$, this implies that $h^{*}+h$ is unbounded, and so, from (7) and (8), $f$ would be unbounded.

7.5. Minorants. Given $f, g \in F$ (and $\geqq 0$ ), we call $f_{1}, g_{1}$ "minorants" of $f, g$ if $f_{1} \leqq f, g_{1} \leqq g$, and $\phi\left(f_{1}\right)=\phi\left(g_{1}\right)$. We shall require the following properties of minorants; the deductions are again straightforward. In this paragraph and the next we again use the convention $\infty-\infty=0$ (cf. 6.2). As before, all elements of $F$ considered are to be non-negative.

(13) If $o<\tau[f] \leqq \tau[g], f$ and $g$ have nonzero minorants.

For, from (4) above (7.3), there exists $h$ such that $[h] \leqq[g]$ and $\phi(h)$ $=\phi(f)$; hence $h \neq 0$, so $g_{1}=\inf (h, g)>0$. We apply (1) to obtain the corresponding $f_{1}$.

(14) A necessary and sufficient condition for $f$ and $g$ to have nonzero minorants is that $\tau[f] \tau[g] \neq 0$.

(From (5), (3) and (13).)

(15) Given $f$ and $g$, there exist $f_{0}$ and $g_{0}$ such that $f_{0} \leqq f, g_{0} \leqq g, \phi\left(f_{0}\right)$ $=\phi\left(g_{0}\right), \tau\left[f_{0}\right]=\tau\left[g_{0}\right]=\tau[f] \tau[g]$, and $\tau\left[f-f_{0}\right] \tau\left[g-g_{0}\right]=o$.

From (14) by "exhaustion" we obtain minorants $f_{0}, g_{0}$ such that $\tau\left[f-f_{0}\right] \tau\left[g-g_{0}\right]=o$; and the remaining properties then follow from (5) and (2).

(16) Given $f$ and $g$, there exists an element $b$ of $A$ such that:

(i) if $a \in A(b), \phi(f \chi(a))=\phi(k)$ for some $k \leqq g \chi(a)$;

(ii) if $a \in A(-b), \phi(g \chi(a))=\phi(l)$ for some $l \leqq f \chi(a)$;

(iii) $b \geqq-\tau[f]$.

Let $f_{0}, g_{0}$ be the minorants of $f, g$ provided by (15), and define $b=-\tau\left[f-f_{0}\right], c=-\tau\left[g-g_{0}\right]$; it is easy to see that $b \bigvee c=e$. From (6) we derive that if $a \in A(b), \phi(f \chi(a))=\phi\left(f_{0} \chi(a)\right)=\phi\left(g_{0} \chi(a)\right)$, in the desired form; and if $a \in A(-b)$, then $a \leqq c$ and the argument is similar.

7.6. Lemma 6. Given $f$, $g$ such that $f$ is bounded and $\tau[g] \leqq \tau[f]$, there exists $a$ unique $h$ such that $h$ is measurable $(A)\left({ }^{30}\right), \tau[h] \leqq \tau[f]$, and $\phi(g)=\phi(f h)$. Further, $[h]=\tau[g]$, and $h \ll \infty$ if and only if $g$ is bounded.

In proving that $h$ exists, we may assume $g \neq 0$. For each positive number $\rho$, we apply (16) to the functions $\rho f$ and $g$, obtaining an element $b_{\rho} \in A$ such

${ }^{\left({ }^{30}\right)}$ That is, the spectrum of $h(1.6)$ consists only of elements of $A$. Equivalently, $h$ belongs to the subspace $F(A)$ of $F(2.4)$. 
that $b_{\rho} \geqq-\tau[f]$. We first show that, for some $\rho>0, b_{\rho}>-\tau[f]$. If not, then $\tau[f] \geqq-b_{\rho}$ for every $\rho$, and so (from (16)ii) $\phi(g)=\phi\left(l_{\rho}\right)$ where $l_{\rho} \leqq \rho f$; and (12) (7.4) then shows that $g=0$, giving a contradiction. Hence there exists $\rho_{1}>0$ such that (with a slight change in notation) $\phi\left\{\rho_{1} f \chi\left(b_{1}\right)\right\}=\phi\left(k_{1}\right)$ where $k_{1} \leqq g$ and $b_{1} \in A$; and since $b_{1} \tau[f] \neq o$ here, we have $k_{1}>0$. If $g-k_{1} \neq 0$, we reapply the argument to $f$ and $g-k_{1}$; and so on, transfinitely. The process must terminate countably, and then $g=\sum k_{\alpha}$, where $\phi\left(k_{\alpha}\right)=\phi\left\{\rho_{\alpha} f \chi\left(b_{\alpha}\right)\right\}$. Write $h_{0}=\sum \rho_{\alpha} \chi\left(b_{\alpha}\right)$, and define $h=h_{0} \chi(\tau[f])$; clearly $h \in F, h \geqq 0, h$ is measurable $(A),[h] \leqq \tau[f]$, and $\phi(h f)=\phi\left(h_{0} f\right)=\phi(g)$. It is easy to see, using (5) and (3), that $\tau[g]=[h]$. If $h_{1}$ also satisfies the condition imposed on $h$ in the lemma, and $h_{1} \neq h$, there must exist a nonzero element $a \in A$, and positive constants $\rho, \sigma$ such that $h_{1} \chi(a) \leqq \rho<\sigma \leqq h \chi(a)$ (or the same with $h_{1}$ and $h$ interchanged). From (6), $\phi(h f \chi(a))=\phi\left(h_{1} f \chi(a)\right)$ where $h_{1} f \chi(a) \leqq \rho f$, and therefore (from (7), (8), (9)) $h f \chi(a)$ is bounded; but $h f \chi(a)=h_{1} f \chi(a)+h_{2}$ where $h_{2} \geqq(\sigma-\rho) f \chi(a)$ $>0$, so that $h f \chi(a)$ is unbounded. This contradiction proves that $h$ is unique.

If $h \ll \infty$, we can write $e=\mathrm{V} a_{n}(n=1,2, \cdots)$ where the $a_{n}$ 's are disjoint elements of $A$ and $(n-1) \chi\left(a_{n}\right) \leqq h \chi\left(a_{n}\right)<n \chi\left(a_{n}\right)$. From (8) and (9), each function $f h \chi\left(a_{n}\right)$ is bounded; hence (10) so is their sum $f h$, and therefore (7) so is $g$. Finally, if $h$ is not finite, there exists a nonzero $a \in A$ such that $h \chi(a)$ $=\infty \chi(a)$; and it readily follows that $g \chi(a)$, and so also $g$, is unbounded.

7.7. Construction of $\phi^{\prime}$. We return to the consideration of possibly negative elements of $F$. Take $f_{0} \in F$ such that $\tau\left[f_{0}\right]=e, f_{0} \geqq 0$, and $f_{0}$ is bounded; from (11), such an $f_{0}$ exists, and we keep $f_{0}$ fixed throughout what follows. For each $g \in F$ such that $g \geqq 0$, apply Lemma 6 (7.6) to $f_{0}$ and $g$, obtaining the unique $h \in F$ such that $h \geqq 0, h$ is measurable $(A)$, and $\phi\left(f_{0} h\right)=\phi(g)$. Write $h=\phi_{0}^{\prime}(g)$; to within an isomorphism, then, $\phi_{0}^{\prime}(g) \in F(A)=F^{\prime \prime}$, say. Let $G_{0}$ be the family of non-negative functions in $F$ which are bounded (in the sense of 7.4). It is easy to verify (using Lemma $1(1.4)$ and 2.1(6)) that $G_{0}$ and $\phi_{0}^{\prime}$ have the properties required in 2.2 , so by Lemma $3(2.2), \phi_{0}^{\prime}$ can be extended to a unique $F^{\prime \prime}$-integral $\phi^{\prime}$ on $F$, defined on a set $G^{\prime} C F$ of "integrable" functions. The construction in 2.2 shows that $\phi^{\prime}(g)=\phi_{0}^{\prime}(g)$ whenever $g \geqq 0$; thus $G^{\prime}$ will be precisely the set of $g \in F$ for which $\phi_{0}^{\prime}\left(g^{+}\right)-\phi_{0}^{\prime}\left(g^{-}\right)$exists, and for each $g \in G^{\prime}$ we have $\phi^{\prime}(g)=\phi_{0}^{\prime}\left(g^{+}\right)-\phi_{0}^{\prime}\left(g^{-}\right)$.

From the uniqueness of $h$ in Lemma 6, we have:

(17) If $f, g \geqq 0$, then $\phi(f)=\phi(g)$ if and only if $\phi^{\prime}(f)=\phi^{\prime}(g)$.

Now, it is an easy consequence of (6) (7.3) that, if $f \geqq 0$ and $a \in A$, then $\phi^{\prime}(f \chi(a))=\phi^{\prime}(f) \chi(a)$. Using (17), we deduce:

(18) If $f \geqq 0, a \in A$, and $\phi^{\prime}(f) \geqq \infty \chi(a)$, then $\phi(f) \geqq \infty \lambda(a)$.

Hence, if $f \in G$, so that $\phi\left(f^{+}\right)-\phi\left(f^{-}\right)$is defined, we must have $\phi^{\prime}\left(f^{+}\right)$ $-\phi^{\prime}\left(f^{-}\right)$defined, and therefore $f \in G^{\prime}$; that is, $G \subset G^{\prime}$. Further, if $f, g \in G$, the statement $\phi(f)=\phi(g)$ is equivalent to the statement $\phi\left(f^{+}+g^{-}\right)=\phi\left(g^{+}+f^{-}\right)$, and thus (from (17)) to the statement $\phi^{\prime}(f)=\phi^{\prime}(g)$. This proves that $\phi$ is a "refinement" of $\phi(2.5)$. 
Finally, let $f \geqq 0$ and $g^{\prime} \in F(A)$ be given such that $0 \leqq g^{\prime} \leqq \phi^{\prime}(f)=h$, say; then, since $\phi\left(f_{0} h\right)=\phi(f)$ and $0 \leqq f_{0} g^{\prime} \leqq f_{0} h$, the homogeneity of $\phi$ gives the existence of $g \in F$ such that $0 \leqq g \leqq f$ and $\phi(g)=\phi\left(f_{0} g^{\prime}\right)$. But the uniqueness condition in Lemma 6 (7.6). now shows that $g^{\prime}=\phi^{\prime}(g)$, so that $\phi^{\prime}$ is fullvalued.

7.8. Uniqueness. Suppose $\phi^{\prime}, \phi^{\prime \prime}$ are two full-valued integrals on $F$, with values in $F^{\prime}$ and $F^{\prime \prime}$ respectively, such that $\phi^{\prime}(f)=\phi^{\prime}(g)$ if and only if $\phi^{\prime \prime}(f)$ $=\phi^{\prime \prime}(g)$, where $f, g \in F$ and $f, g \geqq 0$; we show that $\phi^{\prime}$ and $\phi^{\prime \prime}$ must be isomorphic (in the sense of 2.5). We apply Theorem $9 \beta$ (6.1) to $\phi^{\prime}$ and $\phi^{\prime \prime}$, noting that the corresponding subalgebras $A^{\prime}, A^{\prime \prime}$ of $E$ constructed by 6.2 will be the same for both $\phi^{\prime}$ and $\phi^{\prime \prime}$; this is because an element $x \in E$ is in $A^{\prime}$ (i.e., $x=\pi\left(e, x^{\prime}\right)$ for some $x^{\prime} \in E^{\prime}$ ) if and only if the relations $\phi^{\prime}(f)=\phi^{\prime}(g)$ (where $f, g \geqq 0$ ) and $[g] \leqq x$ imply $[f] \leqq x$. Thus, after isomorphisms on $\phi^{\prime}$ and $\phi^{\prime \prime}$, we can suppose that both have values in $F(A)$, where $A=A^{\prime}=A^{\prime \prime}$. From 7.4 (11), there exists $f_{0} \geqq 0$ such that $\tau\left[f_{0}\right]=e$ and $f_{0}$ is "bounded" for $\phi^{\prime}$, and thus also for $\phi^{\prime \prime}$. Since $\phi^{\prime}$ and $\phi^{\prime \prime}$ are full-valued, it follows that $0 \ll \phi^{\prime}\left(f_{0}\right) \ll \infty$ and $0 \ll \phi^{\prime \prime}\left(f_{0}\right)$ $\ll \infty$; thus, after a further isomorphism of $\phi^{\prime \prime}$, we can make $\phi^{\prime}\left(f_{0}\right)=\phi^{\prime \prime}\left(f_{0}\right)$. Suppose $g \in F, g \geqq 0$, and $\phi^{\prime}(g) \neq \phi^{\prime \prime}(g)$; then we can assume the existence of positive numbers $\rho<\sigma$, and a nonzero $a \in A$, such that $\phi^{\prime}(g) \chi(a) \leqq \rho \phi^{\prime}\left(f_{0}\right) \chi(a)$ $<\sigma \phi^{\prime \prime}\left(f_{0}\right) \chi(a) \leqq \phi^{\prime \prime}(g) \chi(a)$. From Theorem 9, this gives $\phi^{\prime}\left(g_{1}\right) \leqq \phi^{\prime}\left(\rho f_{1}\right)$ $<\phi^{\prime \prime}\left(\sigma f_{1}\right) \leqq \phi^{\prime \prime}\left(g_{1}\right)$, where $g_{1}=g \chi(a)$ and $f_{1}=f_{0} \chi(a)$. Since $\phi^{\prime}$ is full-valued, there exists $h \in F$ such that $0 \leqq h \leqq \rho f_{1}$ and $\phi^{\prime}(h)=\phi^{\prime}\left(g_{1}\right)$; but then $\phi^{\prime \prime}(h)$ $=\phi^{\prime \prime}\left(g_{1}\right)$, which leads at once to a contradiction. Thus $\phi^{\prime}(g)=\phi^{\prime \prime}(g)$ whenever $g \geqq 0$, and so $\phi^{\prime}=\phi^{\prime \prime}$.

7.9. An example. Let $G, G^{\prime}$ denote respectively the sets of "integrable" functions for $\phi$ and $\phi^{\prime}$, where $\phi$ is a homogeneous $F^{\prime}$-integral on $F$ and $\phi^{\prime}$ is its (essentially unique) full-valued refinement. By definition, $G \subset G^{\prime}$. The following example, besides illustrating the general theory, shows that it can happen that $G \neq G^{\prime}$.

Let $S$ be a countably infinite set of points $p_{1}, p_{2}, \cdots$, and let $S^{\prime}$ consist of $S$ together with one more point $p_{0}$. Take $\mathbb{B}$ to be the family of all subsets of $S$, and $\mathcal{N}$ to consist only of the empty set; and similarly for $\mathcal{B}^{\prime}$ and $\mathcal{N}^{\prime}$. Let $F=F(S, B, \mathcal{N}), F^{\prime}=F\left(S^{\prime}, \mathcal{B}^{\prime}, \mathcal{N}^{\prime}\right)$; we define an $F^{\prime}$-integral $\phi$ on $F$ as follows. Take $G$ to be the set of all functions $f$ on $S$ for which the (possibly infinite) sum $\sum_{1}^{\infty} f\left(p_{i}\right)$ exists, and define (for $\left.f \in G\right) \phi(f)$ to be the function $f^{\prime}$ on $S^{\prime}$ given by: $f^{\prime}\left(p_{i}\right)=f\left(p_{i}\right)(i=1,2, \cdots)$, and $f^{\prime}\left(p_{0}\right)=\sum_{1}^{\infty} f\left(p_{i}\right)$. This clearly satisfies the postulates of 2.1 , and $\phi$ is homogeneous since here $\phi(f)$ $=\phi(g)$ implies $f=g$. The "bounded" functions $f(7.4)$ are precisely those (nonnegative) functions which are everywhere finite. It is easy to see that $\tau(x)$ $=x$ here (cf. 7.3), so that $A=E(=\mathbb{B} / \mathcal{N})$; and we may take $f_{0}=1$ in 7.4(11). Thus (as is also obvious directly) the full-valued refinement $\phi^{\prime}$ of $\phi$ is simply the identity mapping of $F$ onto itself. Accordingly $G^{\prime}=F$, but $G \neq F$ since (for example) the function $f$ given by $f\left(p_{n}\right)=(-1)^{n}$ is not in $G$. 


\section{REFERENCES}

1. H. Freudenthal, Teilweise geordnete Moduln, Proc. Nederl. Akad. Wetensch. vol. 39 (1936) pp. 641-651.

2. E. Hille, Functional analysis and semi-groups, Amer. Math. Soc. Colloquium Publications, vol. 31, 1948.

3. L. Kantorovitch, Linear operations in semi-ordered spaces, Rec. Math. (Mat. Sbornik) N.S. vol. 7 (1940) pp. 209-284.

4. D. Maharam, On homogeneous measure algebras, Proc. Nat. Acad. Sci. U.S.A. vol. 28 (1942) pp. 108-111.

5. - The representation of abstract measure functions, Trans. Amer. Math. Soc. vol. 65 (1949) pp. 279-330.

6. - Decompositions of measure algebras and spaces, Trans. Amer. ' 'zih. Soc. vol. 69 (1950) pp. 142-160.

7. H. Nakano, Modern spectral theory, Tokyo, 1950.

8. O. M. Nikodým, Tribus de Boole et fonctions mesurables. Tribu spectrale d'une fonction, C. R. Acad. Sci. Paris vol. 228 (1949) pp. 37-38.

9. S. Saks, Theory of the integral, Warsaw, 1937.

10. M. H. Stone, Boundedness properties in function-lattices, Canadian Journal of Mathematics vol. 1 (1949) pp. 176-186.

MANChester, ENGLAND. 